\title{
Novel maximum likelihood approach for passive detection and localisation of multiple emitters
}

\author{
Marcel Hernandez
}

\begin{abstract}
In this paper, a novel target acquisition and localisation algorithm (TALA) is introduced that offers a capability for detecting and localising multiple targets using the intermittent "signals-of-opportunity" (e.g. acoustic impulses or radio frequency transmissions) they generate. The TALA is a batch estimator that addresses the complex multi-sensor/multi-target data association problem in order to estimate the locations of an unknown number of targets. The TALA is unique in that it does not require measurements to be of a specific type, and can be implemented for systems composed of either homogeneous or heterogeneous sensors. The performance of the TALA is demonstrated in simulated scenarios with a network of 20 sensors and up to 10 targets. The sensors generate angle-of-arrival (AOA), time-of-arrival (TOA), or hybrid AOA/TOA measurements. It is shown that the TALA is able to successfully detect $83-99 \%$ of the targets, with a negligible number of false targets declared. Furthermore, the localisation errors of the TALA are typically within $10 \%$ of the errors generated by a "genie" algorithm that is given the correct measurement-to-target associations. The TALA also performs well in comparison with an optimistic Cramér-Rao lower bound, with typical differences in performance of 10-20\%, and differences in performance of $40-50 \%$ in the most difficult scenarios considered. The computational expense of the TALA is also controllable, which allows the TALA to maintain computational feasibility even in the most challenging scenarios considered. This allows the approach to be implemented in time-critical scenarios, such as in the localisation of artillery firing events. It is concluded that the TALA provides a powerful situational awareness aid for passive surveillance operations.
\end{abstract}

Keywords: Passive detection and localisation, Multi-sensor/multi-target data association, Maximum likelihood estimation, Gauss-Newton gradient descent, Cramér-Rao lower bound, Time-of-arrival measurements

\section{Introduction}

Recently, there has been great interest in the detection, localisation and tracking of noncooperative targets using passive sensors that exploit the "signals-of-opportunity" generated by such targets (e.g. see [1] and references therein). Typical applications include detection and localisation of weapon firing events $[2,3]$ and localisation in wireless communication systems $[4,5]$, non-line-ofsight (e.g. urban) environments [6], and in search/rescue operations [7].

Passive surveillance has the advantage of covertness, and passive sensors are typically smaller, cheaper, and lower power than their active counterparts. This allows

Correspondence: marcel.I.hernandez@gmail.com

Hernandez Technical Solutions Ltd, 38A Lower Chase Road, Malvern, Worcestershire WR14 2BZ, United Kingdom passive sensors to be utilised in scenarios that would preclude the deployment of active sensors (e.g. such as in remote operations). Passive measurement exploitation has a long history, beginning with angle-of-arrival (AOA) emitter localisation [8]. Other commonly used passive measurements include time-of-arrival (TOA), time difference of arrival (TDOA), frequency of arrival (FOA), and combinations thereof (e.g. again, see [1] and references therein).

Due to the intermittent nature of target signals in passive surveillance operations (e.g. artillery firings may occur in short bursts only every few days), recursive estimation techniques (e.g. such as the extended Kalman filter [9]) may be ineffective, because the interval between signals is too large to allow persistent target tracking. Therefore, it is common to perform batch estimation in 
order to detect and localise target emitters, exploiting all measurements generated by the sensors within a time window, e.g. $[2,4,5,10-13]$. The time window must be sufficiently large to account for target signal propagation delays between the sensors.

Previous work on passive emitter geo-location has largely concentrated on the case of a single target, thereby avoiding the problem of associating measurements to targets. For example, maximum likelihood (ML) approaches have been developed for single target localisation using either TOA measurements [14], range measurements generated by a multi-static passive radar [15], or TDOA measurements [16]. Target localisation using hybrid measurements has also been performed, with least squares (e.g. $[4,5])$ and ML (e.g. [11, 12]) approaches developed in order to localise a single emitter using hybrid AOA/TDOA measurements.

To-date, only a small number of papers have considered the problem of localising multiple emitters $[2,10,13,17-20]$. Some of these papers have addressed simplified scenarios with either perfect measurements $[17,18]$ or a known number of emitters [13]. The remaining papers have developed techniques specifically for a TOA measurement model $[2,10,19,20]$, and these approaches cannot be easily modified to deal with other measurement models (e.g. hybrid AOA/TOA measurements). Furthermore, as noted in [10], there remains a requirement to generalise existing approaches in order that they can exploit measurements generated by a network of heterogeneous sensors.

The target acquisition and localisation algorithm (TALA) introduced herein addresses the complex multisensor/multi-target data association problem, in order to detect and localise an unknown number of target signals/events (e.g. such as acoustic impulses generated by artillery firings or intermittent radio frequency transmissions). The TALA is a batch estimator, and its novelty lies in the mechanism by which it circumnavigates the need to perform global multi-sensor/multi-target data association, e.g. as necessary in [20], thereby allowing the TALA to maintain computational feasibility, even for large-scale problems. Furthermore, unlike existing approaches (e.g. $[2,10,19,20])$, the TALA does not require measurements to be of a specific type (i.e. TOA measurements) and can be implemented for systems composed of either homogeneous or heterogeneous sensors.

Specifically, the TALA initially performs nearest neighbour data association (e.g. see [21]) on a measurementby-measurement basis, allowing each measurement to be associated with multiple hypothesised target locations. The algorithm formulates a set of potential target location hypotheses, and then performs Gauss-Newton (G-N) gradient descent (e.g. [24]) to provide maximum likelihood estimates of these locations, before a final downselection step, ensures that each measurement is associated with no greater than one estimate.

This mechanism for handling the complex multisensor/multi-target association problem, based on manipulating multiple competing hypotheses, removes the need to perform global data association (which can be a computationally prohibitive combinatorial optimisation, e.g. see [22]), and is analogous to the track-oriented multiple hypothesis tracking (TOMHT) methodology introduced in [22]. However, unlike the TOMHT, the TALA is a non-recursive (i.e. "one-shot") approach, rather than the update of an existing target set.

The remainder of this paper is organised as follows. In Section 2, the TALA is described. In Section 3, details are provided of the two measures of optimal estimation performance that are used to baseline the performance of the TALA. The first measure is the Cramér-Rao lower bound [23], and the second is a "genie" TALA that is given the correct measurement-to-target associations. In Section 4, simulation results are presented for scenarios in which all sensors provide hybrid AOA/TOA measurements, and scenarios in which $50 \%$ of the sensors provide only AOA measurements, with the remaining sensors providing only TOA measurements. A discussion is presented in Section 5 with conclusions following in Section 7. Section 6 provides details of recommendations for future work. Finally, Appendix A provides details of the methodology for determining initial candidate target locations using the AOA and distance difference of arrival (DDOA) measurements available in the simulations.

\section{Target acquisition and localisation algorithm}

\subsection{Overview}

The TALA is a batch estimation algorithm that utilises all measurements generated within a time window by an array of sensors, in order to detect and localise an unknown number of target events (i.e. intermittent signals, such as acoustic impulses or radio frequency transmissions). Initially, the TALA generates "candidate" target locations, and then performs "soft" nearest neighbour data association (e.g. [21]), allowing each measurement to be associated with more than one candidate location. This approach removes the need to perform global multisensor/multi-target data association, e.g. as necessary in [20], thereby maintaining computational feasibility, even for large scale problems.

Using the measurements associated with each candidate location, ML estimation is then performed in order to localise each potential target. The ML estimation problem cannot be solved analytically, and an iterative G-N approach (e.g. [24]) is used to solve an equivalent nonlinear least squares problem. The G-N approach performs iterative gradient descent, and in order to combat 
potential divergence, line search and randomisation are used to ensure that each iteration increases the value of the likelihood. It is noted that alternative techniques, such as the Newton-Raphson (N-R) approach (e.g. [25]) or the Levenberg-Marquardt algorithm [26, 27], could also be used to perform the gradient descent and may offer similar performance.

\subsection{Summary of the main steps}

The main steps in the TALA are as follows:

\section{Step 1: Determine initial candidate locations}

- If possible (generally only in two-dimensional emitter geo-location), determine the intersection between measurements generated by each pair of sensors.

- More generally, determine a candidate location that minimises a Mahalanobis-based distance metric using measurements generated by each pair of sensors.

- These points form the initial candidate (target) location set.

- In cases for which performing measurement intersection or Mahalanobis distance minimisation is problematic/impossible, initial candidate locations should be randomly sampled within the surveillance region.

2. Step 2: Associate measurements and determine likelihood for each candidate location

- Determine the measurement from each sensor that has the greatest individual likelihood (or equivalently the smallest Mahalanobis distance) for each candidate location.

- This measurement is associated with the location provided that the individual likelihood is greater than a pre-specified threshold value.

- Each measurement is allowed to be associated with more than one candidate location.

- The overall likelihood of each candidate location is calculated using all of the associated measurements.

\section{Step 3: Candidate location deletion}

- The number of candidate locations can be large.

- To reduce the computational expense of the algorithm, at this stage, some of the candidate locations are deleted.

- A candidate location is deleted if it either has too few measurements associated with it or if it shares identical associations with another candidate target location that has a greater overall likelihood.
- Optionally, the candidate location is deleted if it shares any associations with another candidate target location that has a greater overall likelihood.

\section{Step 4: Maximum likelihood estimation}

- Using the candidate locations retained from Step 3, plus the measurements associated with each location, determine ML estimates via an iterative G-N approach.

- Optionally, measurement reassociation may be performed on each iteration of the G-N algorithm.

\section{Step 5: Final downselection/outputs}

- Perform downselection to ensure that each measurement is associated with only one ML estimate.

An illustrative example of the TALA is shown in Figs. 1 and 2 .

\subsection{Step 1: Determine initial candidate locations}

An $N$ sensor system is considered. Let $n(i)$ denote the number of measurements, each of dimensionality $d_{i}$, generated by sensor $i$. Let $z(i, j)$ denote the $j$-th measurement generated by sensor $i$. It is assumed that target-generated measurements are corrupted by additive Gaussian noise ${ }^{1}$. Hence, for a target located at coordinates $X \in \mathbb{R}^{3}$, each target-generated measurement at sensor $i$ is given as follows:

$$
z(i, .)=\boldsymbol{f}(X ; i)+\boldsymbol{e}(i)
$$

where $\boldsymbol{f}(\boldsymbol{X} ; i) \triangleq\left(f_{1}(\boldsymbol{X} ; i) \ldots f_{d_{i}}(\boldsymbol{X} ; i)\right)^{\prime}$. Each measurement error $\boldsymbol{e}(i) \sim \mathcal{N}\left(0, \boldsymbol{\Sigma}_{i}\right)$, with $\boldsymbol{\Sigma}_{i}$ denoting the error covariance of each target-generated measurement at sensor $i$.

The first step in the TALA is to generate a set of initial candidate location hypotheses, with these hypotheses then manipulated in order to determine ML estimates of the locations of an unknown number of targets. Therefore, it would seem prudent to choose candidate locations that are consistent with the measurements. To this end, the following methodology is used to generate initial candidate locations:

1. If the focal problem is concerned with the geo-location of targets within a two-dimensional region (e.g. the geo-location of ground-based targets within a geographically flat region), initial candidate locations can be determined as the intersection of each pair of measurements (if such an intersecting 


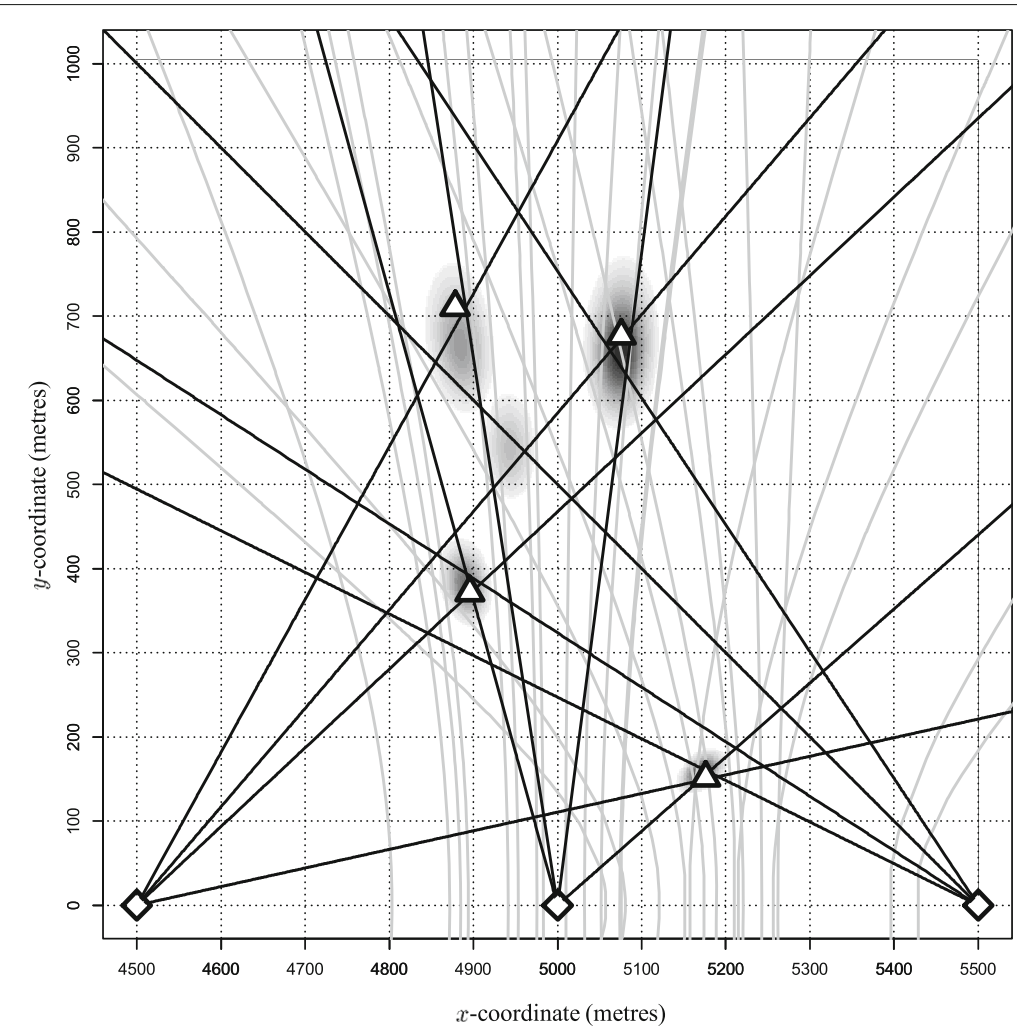

Fig. 1 An exemplar scenario with four target events and three sensors that provide hybrid AOA/TOA measurements. Shown are target events (marked by triangles) and the sensors (marked by diamonds); along with AOA measurements (black lines) and DDOA measurement hyperbolae (grey lines). Also shown is the likelihood map, with dark regions showing a normalised overall measurement likelihood close to unity, and white regions showing a normalised overall measurement likelihood close to zero. It is noted that this likelihood map is not calculated by the TALA and is shown for illustrative purposes only

point exists). In later simulations, the intersections between pairs of AOA measurements and pairs of DDOA measurements are used to generate initial candidate locations.

2. For more complex applications in which measurement intersection cannot be performed (e.g. three-dimensional target geo-location, in which case the measurements are extremely unlikely to intersect because of the presence of measurement errors), for each pair of measurements $\hat{z} \triangleq\left(\boldsymbol{z}\left(i_{1}, .\right)^{\prime} \boldsymbol{z}\left(i_{2}, .\right)^{\prime}\right)^{\prime}$, for $i_{1} \neq i_{2}$; a candidate location $\boldsymbol{X}_{c}$ can be determined by minimising the Mahalanobis distance between $\hat{z}$ and $\hat{\boldsymbol{f}}(\boldsymbol{X}) \triangleq\left(\boldsymbol{f}\left(\boldsymbol{X} ; i_{1}\right)^{\prime} \boldsymbol{f}\left(\boldsymbol{X} ; i_{2}\right)^{\prime}\right)^{\prime}$, i.e.

$$
\boldsymbol{X}_{c}=\underset{\boldsymbol{X} \in \mathbb{R}^{3}}{\arg \min }\left[\boldsymbol{\epsilon}(\boldsymbol{X})^{\prime} \hat{\boldsymbol{\Sigma}}^{-1} \boldsymbol{\epsilon}(\boldsymbol{X})\right]
$$

where $\boldsymbol{\epsilon}(\boldsymbol{X}) \triangleq(\hat{\boldsymbol{z}}-\hat{\boldsymbol{f}}(\boldsymbol{X}))$; and $\hat{\boldsymbol{\Sigma}}$ is the error covariance of the measurement $\hat{z}$.

It may be necessary to limit the number of candidate locations by not considering all combinations of sensor measurements in determining the intersections (in two-dimensional applications) or minimising (2) (in three-dimensional applications). Moreover, in threedimensional geo-location applications, the optimisation in Eq. (2) may not be straightforward, and it may be more efficient to randomly select candidate locations within the surveillance region.

\subsection{Step 2: Associate measurements and determine likelihood for each candidate location}

It is assumed that for each sensor, a maximum of one measurement is generated by each target in the time window under consideration. Furthermore, for each candidate target location $\boldsymbol{X}$, and each sensor $i$ :

1. The index $a(X ; i) \in\{1, \ldots, n(i)\}$ of the measurement that is associated is the one with the largest individual likelihood, i.e. nearest neighbour data association is performed (e.g. see [21]).

2. If every measurement generated by a sensor has an individual likelihood that is less than $100 \xi \%$ of the maximum value $l_{i}(\max )$, then no measurement from that sensor is associated with the location ${ }^{2}$. The 

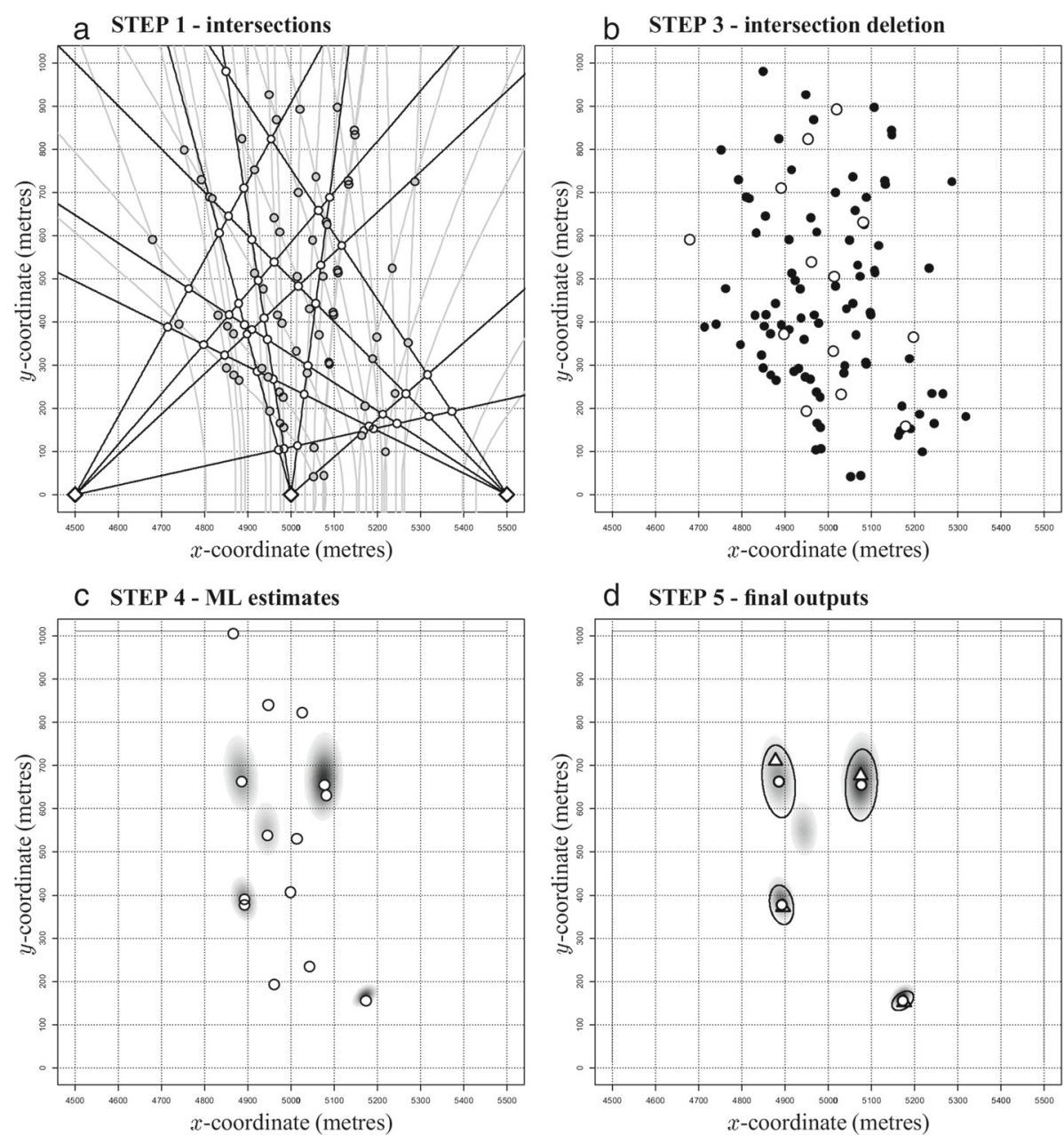

Fig. 2 Demonstration of the TALA for the scenario with four targets. a Step 1: intersection of each pair of AOA measurements (white circles) and each pair of DDOA measurement hyperbolae (grey circles). b Step 3: intersections that are retained (white circles), and intersections that are deleted (black circles; deletion criterion 3 is not used). c Step 4: ML estimates. d Step 5: downselected ML estimates (circles), and the true target locations (triangles). Each ellipse shows the 5 standard deviation error covariance associated with each ML estimate

threshold $\xi \in[0,1]$ is pre-specified ${ }^{3}$. It is noted that this approach is equivalent to gating the

measurement Mahalanobis distance with a threshold $g=\sqrt{-2 \ln \xi}$.

Therefore, $a(X ; i)$ is given as follows:

$a(X ; i) \triangleq \begin{cases}\underset{j=1, \ldots, n(i)}{\arg \max } l_{i}(X ; j) & \text { if } \max _{j=1, \ldots, n(i)} l_{i}(X ; j) \geq \xi \times l_{i}(\max ) \\ -1 & \text { otherwise }\end{cases}$

where $l_{i}(X ; j)$ denotes the individual likelihood at sensor $i$ as a result of associating the $j$-th measurement $z(i, j)$ with candidate target location $\boldsymbol{X}$. For the measurement model
(1), this individual likelihood is given as follows:

$$
\begin{aligned}
l_{i}(\boldsymbol{X} ; j)= & \frac{1}{(2 \pi)^{d_{i} / 2} \operatorname{det}\left(\boldsymbol{\Sigma}_{i}\right)^{1 / 2}} \exp \left(-\frac{1}{2}[\boldsymbol{z}(i, j)\right. \\
& \left.-\boldsymbol{f}(\boldsymbol{X} ; i)]^{\prime} \boldsymbol{\Sigma}_{i}^{-1}[\boldsymbol{z}(i, j)-\boldsymbol{f}(\boldsymbol{X} ; i)]\right)
\end{aligned}
$$

It is noted that $a(X ; i)=-1$ denotes that no measurement from sensor $i$ is associated with the location $X$.

The overall measurement likelihood is then given as follows:

$$
\begin{aligned}
L(\boldsymbol{X})= & \frac{1}{(2 \pi)^{D / 2} \operatorname{det}(\boldsymbol{\Sigma})^{1 / 2}} \exp \left\{-\frac{1}{2}[\boldsymbol{Z}\right. \\
& \left.-\boldsymbol{f}(\boldsymbol{X})]^{\prime} \boldsymbol{\Sigma}^{-1}[\boldsymbol{Z}-\boldsymbol{f}(\boldsymbol{X})]\right\}
\end{aligned}
$$


where:

$$
\begin{aligned}
N_{a} \triangleq & \text { total number of measurements associated with } \\
& \text { the location } \boldsymbol{X} \\
& =\sum_{i=1}^{N} \sum_{a(X ; i)>-1} 1 \\
Z & \triangleq \text { concatenated vector of associated measurements } \\
& =\left(\boldsymbol{z}(1, a(X ; 1))^{\prime} \ldots \boldsymbol{z}\left(N_{a}, a\left(X ; N_{a}\right)\right)^{\prime}\right)^{\prime}
\end{aligned}
$$

(with the sensor indices reordered to $1, \ldots, N_{a}$ )

$$
\begin{aligned}
\boldsymbol{f}(\boldsymbol{X})= & \left(\boldsymbol{f}(\boldsymbol{X} ; 1)^{\prime} \ldots \boldsymbol{f}\left(\boldsymbol{X} ; N_{a}\right)^{\prime}\right)^{\prime} \\
D= & \text { dimensionality of the concatenated vector of } \\
& \text { all associated measurements } \\
= & \sum_{i=1}^{N} \sum_{a(X ; i)>-1} d_{i} \\
\boldsymbol{\Sigma}= & \left(\begin{array}{cccc}
\boldsymbol{\Sigma}_{1} & \boldsymbol{\Sigma}_{2,1} & \ldots & \boldsymbol{\Sigma}_{N_{a}, 1} \\
\boldsymbol{\Sigma}_{1,2} & \boldsymbol{\Sigma}_{2} & \ddots & \vdots \\
\vdots & \ddots & \ddots & \boldsymbol{\Sigma}_{N_{a}, N_{a}-1} \\
\boldsymbol{\Sigma}_{1, N_{a}} & \ldots & \boldsymbol{\Sigma}_{N_{a}-1, N_{a}} & \boldsymbol{\Sigma}_{N_{a}}
\end{array}\right) \\
\boldsymbol{\Sigma}_{i, j} \triangleq & \text { correlation between the measurements at } \\
& \text { sensors } i \text { and } j
\end{aligned}
$$

It is noted that if the measurements from all sensors are uncorrelated (i.e. $\boldsymbol{\Sigma}_{i, j}=0$, for all $i, j$ ), the overall measurement likelihood at each candidate location $X$ is given by the product of the individual likelihood values of the associated measurements, i.e.

$$
L(X)=\prod_{i=1}^{N} \prod_{a(X ; i)>-1} l_{i}(X ; a(X ; i))
$$

More importantly, in this case, the ensemble of measurements that satisfy Eq. (3), for $i=1, \ldots, N$, also maximises the overall measurement likelihood.

There is no practical reason why the nearest neighbour data association approach cannot be used if the measurements from different sensors are correlated. However, it should be noted that the resulting measurement set is not guaranteed to be close to optimal in maximising the overall measurement likelihood. In such cases, performing measurement reassociation during gradient descent (see Section 2.6.3) may be helpful in correctly resolving the complex multi-sensor/multi-target data association problem.
An exemplar likelihood map is shown in Fig. 1. It is noted that this map is shown for illustration only. The reader is reminded that the TALA calculates the likelihood only at the initial candidate locations and at the locations determined on subsequent iterations of the gradient descent algorithm.

\subsection{Step 3: Candidate location deletion}

Clearly, the number of candidate locations can be large. To reduce the computational expense of the algorithm, at this stage, some of the candidate locations are deleted. A candidate location is deleted if any of the following are true.

1. Deletion criterion 1: The candidate location does not have at least $\mu P_{d} N$ measurements associated with it (i.e. it is not consistent enough with the data). This value is set by noting that the average number of measurements generated by each target is $P_{d} N$ for a system with $N$ sensors, and with a probability $P_{d}$ that each target is detected by each sensor. In simulations, a value of $\mu=0.5$ was shown to produce excellent results.

2. Deletion criterion 2: The candidate location has exactly the same measurements associated with it as another candidate location that has greater overall likelihood.

3. Deletion criterion 3 (optional): The candidate location has one or more measurements associated with it that are also associated with another candidate location that has greater overall likelihood. The procedure for implementing this deletion criterion is as follows:

(a) The overall likelihood is calculated for each candidate location, using the procedure described on Step 2 of the algorithm.

(b) The candidate location with the greatest overall likelihood is accepted as a potential target location.

(c) Recursively, consider the candidate location with next greatest likelihood. If this candidate location does not share any associations with any of the previously accepted candidate locations it is also accepted as a potential target location, otherwise it is deleted.

Deletion criterion 3 has the advantage of significantly reducing the number of candidate locations that need to be manipulated, and this can significantly reduce the computational expense of the algorithm. The disadvantage is that by deleting candidate locations at this early stage, the TALA has a reduced probability of detecting all target events. This criterion therefore compromises estimator performance for increased computational speed. 
In Fig. 2b, the results of the intersection deletion step are shown for the exemplar scenario. It is noted that deletion criterion 3 is not used in this example.

\subsection{Step 4: Maximum likelihood estimation}

\subsubsection{Background - standard Gauss-Newton approach}

Consider the set of $N_{a}$ measurements associated with a candidate location, calculated via Eq. (3). The ML estimate $\hat{\boldsymbol{X}}_{M L E}$ of the target location, based on these measurements is given as follows:

$$
\begin{aligned}
\hat{\boldsymbol{X}}_{M L E} & =\underset{\boldsymbol{X}}{\arg \max } L(\boldsymbol{X}) \\
& =\underset{\boldsymbol{X}}{\arg \min }\left[[\boldsymbol{Z}-\boldsymbol{f}(\boldsymbol{X})]^{\prime} \boldsymbol{\Sigma}^{-1}[\boldsymbol{Z}-\boldsymbol{f}(\boldsymbol{X})]\right]
\end{aligned}
$$

with $\boldsymbol{Z}, \boldsymbol{f}(\boldsymbol{X})$, and $\boldsymbol{\Sigma}$ given in Eqs. (9), (10), and (13) respectively.

The non-linear least squares problem (17) can be solved using the G-N approach (e.g. [24]). The G-N approach performs iterative gradient descent, starting with an initial estimate $\boldsymbol{X}_{0}$. It generates a sequence of estimates as follows:

$$
\boldsymbol{X}_{k+1}=\boldsymbol{X}_{k}-\boldsymbol{\delta}_{k}
$$

where the full "Newton step" $\boldsymbol{\delta}_{k}$ is given as follows:

$$
\boldsymbol{\delta}_{k}=\left[\boldsymbol{F}\left(\boldsymbol{X}_{k}\right)^{\prime} \boldsymbol{\Sigma}^{-1} \boldsymbol{F}\left(\boldsymbol{X}_{k}\right)\right]^{-1} \boldsymbol{F}\left(\boldsymbol{X}_{k}\right)^{\prime} \boldsymbol{\Sigma}^{-1}\left[\boldsymbol{Z}-\boldsymbol{f}\left(\boldsymbol{X}_{k}\right)\right]
$$

The Hessian matrix $\boldsymbol{F}\left(\boldsymbol{X}_{k}\right)$ is given as follows:

$\boldsymbol{F}\left(\boldsymbol{X}_{k}\right)=\left(\nabla_{\boldsymbol{X}_{k}} \boldsymbol{f}\left(\boldsymbol{X}_{k}\right)^{\prime}\right)^{\prime}=\left(\nabla_{\boldsymbol{X}_{k}} \boldsymbol{f}\left(\boldsymbol{X}_{k} ; 1\right)^{\prime} \ldots \nabla_{\boldsymbol{X}_{k}} \boldsymbol{f}\left(\boldsymbol{X}_{k} ; N_{a}\right)^{\prime}\right)^{\prime}$

where $\nabla_{X_{k}}$ is the first-order partial derivative operator with respect to $\boldsymbol{X}_{k} \in \mathbb{R}^{3}$.

If the iterative scheme given in Eq. (18) converges, it will do so to a stationary point, thereby providing a ML estimate. However, convergence is not guaranteed and is highly dependent on the proximity of the initial estimate $X_{0}$ to the stationary value.

\subsubsection{Implementation - Gauss-Newton approach with an adaptive step size}

In light of the potential for the G-N approach to diverge, the implementation herein allows steps smaller than, and in the opposite direction to, the full "Newton step", whilst attempting to maximise the increase in the overall measurement likelihood on each iteration. Specifically, the G-N approach is initialised with each initial candidate location. On each subsequent iteration, the location is modified as follows:

$$
\boldsymbol{X}_{k+1}=\boldsymbol{X}_{k}+\boldsymbol{\Lambda}_{k}
$$

where, either:
- $\boldsymbol{\Lambda}_{k}$ is the increment from the set $\left\{\alpha \boldsymbol{\delta}_{k} / m: \alpha=-m, \ldots,-1,1, \ldots, m\right\}$ that results in the greatest increase in the overall measurement likelihood; where $\boldsymbol{\delta}_{k}$ is the full Newton step (19) and $m$ is a pre-specified positive integer;

or, if no step from the above set increases the overall measurement likelihood:

- $\boldsymbol{\Lambda}_{k}$ is a step in a randomly generated direction (i.e. drawn from a Uniform distribution on $[-\pi, \pi]$ ) of magnitude $\delta_{M}$ (nominally, $\delta_{M}=200$ metres). This random step is accepted if it increases the overall measurement likelihood.

The G-N approach is terminated if either:

1. A total of 20 random steps have been attempted.

2. The magnitude of each component of the gradient of the normalised sum-of-squared errors $(\text { GNSSE })^{4}$ is smaller than a pre-specified value (nominally $10^{-3}$ ). Only in this case is successful convergence to a ML estimate deemed to have been achieved.

This "line search" adaptation of the G-N approach is similar to the line search approach detailed in Section 9.7 in [25]. In Fig. 2c, ML estimates calculated using the G-N approach are shown for the exemplar scenario.

\subsubsection{Reassociation during gradient descent}

In scenarios in which the measurement errors are large, each initial candidate location (e.g. generated from the intersection of a pair of measurements) may be distant from the ML estimate. In such cases, the measurements associated with the initial candidate location may not be the nearest to each of the subsequent iterates, $\boldsymbol{X}_{k}, k=$ $1,2, \ldots$, of the G-N algorithm.

Motivated by this, in cases in which the measurements are inaccurate, reassociation can be performed after each iteration of the G-N approach. That is, having determined iterate $\boldsymbol{X}_{k}$, reassociation is performed, and the measurements associated with location $\boldsymbol{X}_{k}$ are used to determine the next increment $\boldsymbol{\delta}_{k}$, and next iterate $\boldsymbol{X}_{k+1}$.

Performing reassociation can significantly improve performance when measurement errors are large. However, this is at the cost of (i) increasing the computational expense of the algorithm and (ii) making the algorithm less likely to converge to a ML estimate, hence reducing the number of target events located.

\subsection{Step 5: Final downselection/outputs}

Having determined the ML estimates on Step 4, downselection is performed in order to ensure that each measurement is associated with no greater than one ML estimate. The procedure for performing this downselection is exactly the same as given in the optional deletion 
criterion 3 on Step 3. It is noted that if the optional criterion is performed on Step 3, and provided that reassociation is not performed during the gradient descent on Step 4 , then this downselection has already been performed.

A final downselection step also deletes estimates that lie within the sensor perimeter. Such estimates are rare, but can occur because of incorrect associations, or convergence to the wrong point of intersection of the associated measurements.

The remaining ML estimates provide estimates of the target event locations. The approximate error covariance (denoted $\mathcal{C}\left(X^{\star}\right)$ ) of each estimate $X^{\star}$ is given by the inverse of the observed Fisher information matrix [28]. This covariance is as follows:

$$
\mathcal{C}\left(X^{\star}\right) \approx\left[F\left(X^{\star}\right)^{\prime} \Sigma^{-1} F\left(X^{\star}\right)\right]^{-1}
$$

The matrix $\boldsymbol{\Sigma}$ is again given by Eq. (13); and the matrix $\boldsymbol{F}($.) is given by Eq. (20). In Fig. 2d, the final outputs of the target localisation algorithm are shown for the exemplar scenario.

\section{Measures of optimal estimation performance}

\subsection{Cramér-Rao lower bound}

Let $X$ denote the location of a target event. The CramérRao lower bound (CRLB) (e.g. [23]) provides a bound on the mean square error (MSE) of any (unbiased) estimator $\hat{X}$ of $X$, i.e.

$$
\mathbb{E}_{\boldsymbol{Z} \mid \boldsymbol{X}}\left[(\boldsymbol{X}-\hat{\boldsymbol{X}})(\boldsymbol{X}-\hat{\boldsymbol{X}})^{\prime}\right] \geq \boldsymbol{J}(\boldsymbol{X})^{-1} \triangleq \mathrm{CRLB}
$$

where $\boldsymbol{J}(\boldsymbol{X})$ is the Fisher information matrix (FIM) and $\mathbb{E}_{Z \mid X}$ denotes mathematical expectation with respect to the measurement vector $Z$, given $X$. The CRLB provides a bound on the performance of any unbiased target localisation algorithm.

In the case in which there can be missed detections (i.e. $P_{d}<1$ ), it is straightforward to show that for a realisation of target detections at each of the sensors, the conditional FIM is given as follows:

$$
\tilde{\boldsymbol{J}}(\boldsymbol{X})=\tilde{\boldsymbol{F}}(\boldsymbol{X})^{\prime} \tilde{\boldsymbol{\Sigma}}^{-1} \tilde{\boldsymbol{F}}(\boldsymbol{X})
$$

where $\tilde{\boldsymbol{F}}$ is the Hessian (20) determined only for sensors that have detected the target. The matrix $\tilde{\boldsymbol{\Sigma}}$ is the associated measurement error covariance. The CRLB is then approximated as follows:

$$
\mathrm{CRLB} \approx \frac{1}{n_{s}} \sum_{r=1}^{n_{s}} \tilde{\boldsymbol{J}}_{r}(\boldsymbol{X})^{-1}
$$

where $n_{s}$ is the number of measurement sequence realisations considered, and $\tilde{\boldsymbol{J}}_{r}(\boldsymbol{X})$ is the conditional FIM for measurement sequence realisation $r$. This is referred to the "enumeration" bound [29] and has been shown to be the least optimistic formulation of the CRLB [30] for the case $P_{d}<1$.

The CRLB location root mean square error (RMSE) is then given as follows:

CRLB location RMSE $=\sqrt{\mathrm{CRLB}_{11}+\mathrm{CRLB}_{22}+\mathrm{CRLB}_{33}}$

where $\mathrm{CRLB}_{i i}$ denotes the $i$-th diagonal entry of the CRLB (this provides a MSE bound for the estimation of the $i$-th coordinate of the target event).

In the simulations that follow, for each target location $X$, a single measurement sequence realisation is considered (i.e. $n_{s}=1$ ). The overall CRLB is then calculated by averaging the value of the CRLB for each of the target locations, with its associated measurement sequence realisation, used in the simulations.

It is noted that this CRLB formulation can still be optimistic because the bound is calculated independently for each target event. The formulation therefore does not take into account the difficulty of associating measurements between targets, in multiple event scenarios. Formally, this difficulty is quantified via an information reduction matrix, which is extremely complex and can only be calculated via Monte Carlo integration. For full details of the approach, the reader is referred to [31].

In later simulations, there is also the potential for spurious false alarm (i.e. "ghost") measurements to occur. It is therefore noted that the CRLB formation utilised herein does not take into account the impact of these false measurements. The reader is referred to [32] for details of how to adjust the CRLB in the presence of false alarms.

\section{2 "Genie" TALA with the measurement-to-target associations given}

In light of the potential optimism of the CRLB formulation presented in the previous section, a second performance measure is provided in order to help quantify the optimality of the TALA. This second performance measure is a "genie"-based algorithm, in the spirit of [10], that is given the correct measurement-to-target associations.

Specifically, for each target, the genie algorithm attempts to determine a ML estimate as follows:

1. The G-N approach (given on Step 4 of the TALA) is used in an attempt to determine a ML estimate, using the measurements generated by the target.

2. The G-N approach is initialised using the intersection of two of the target-generated measurements, if such an intersection exists. Otherwise, an initial location is randomly generated, as in step 3 below.

3. If the $\mathrm{G}-\mathrm{N}$ approach fails to converge to a $\mathrm{ML}$ estimate, an initial candidate location is randomly 
generated within the surveillance region, and the G-N approach is rerun. A maximum of 20 randomly generated initial locations are attempted.

This performance measure is referred to as the "genie" TALA (gTALA). Clearly, the gTALA is likely to provide a bound on the optimal performance of the TALA because it avoids the potentially complex problem of associating measurements to targets. However, a performance bound is not guaranteed.

\section{Simulations}

\subsection{Scenarios considered}

A sensor system, comprising of an array of 20 microphones, is deployed in order to detect and localise acoustic artillery firing events that occur within a two-dimensional geographical region (see Fig. 3).

Three scenarios are considered, given as follows:

1. Scenario 1: Target events occur at different times, with the time instance of each event sampled from a uniform distribution on $[0,100]$ seconds. Each sensor provides hybrid AOA/TOA measurements.

2. Scenario 2: Target events occur simultaneously. Again, each sensor provides hybrid AOA/TOA measurements.
3. Scenario 3: Target events occur simultaneously. Fifty percent of the sensors provide only AOA measurements, with the remaining sensors providing only TOA measurements.

In all scenarios, the number of false measurements generated by each sensor has a Poisson distribution with mean $1.5 \times 10^{-7}$ per square metre of the surveillance region. As a result, there are an average of 2.2 false measurements per sensor. Each false measurement is generated at a random location uniformly distributed within the surveillance region. False alarms at different sensors are generated at different locations within the surveillance region. The parameter settings are summarised in Table 1.

\subsection{Measurement generation}

\subsubsection{AOA and TOA measurements}

Each sensor generates two-dimensional measurements of AOA (i.e. azimuth) and/or TOA of target events. If an event occurs at Cartesian coordinates $\boldsymbol{X}_{k} \triangleq\left(x_{k}, y_{k}\right)$ at time $t_{k}$, target-generated measurements (when they exist) are given as follows:

$$
\begin{aligned}
& \mathrm{AOA} \triangleq \theta(.)=\tan ^{-1}\left(\frac{y_{k}-y_{S}}{x_{k}-x_{S}}\right)+e_{\theta}(.) \\
& \mathrm{TOA} \triangleq t(.)=t_{k}+d\left(\boldsymbol{X}_{k}\right) / c+e_{t}(.)
\end{aligned}
$$

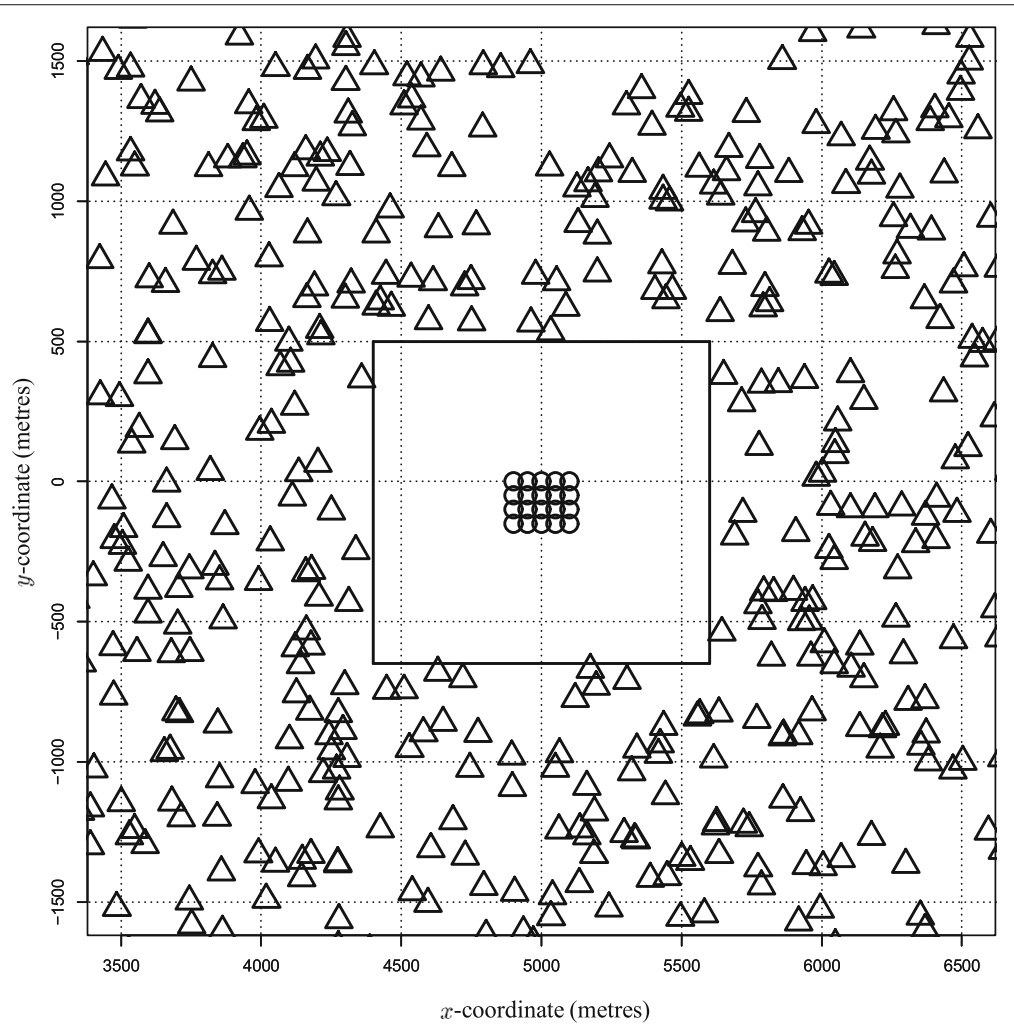

Fig. 3 Surveillance scenario. Shown are the sensor locations (circles) and randomly generated target event locations (triangles). Target events are not generated within $500 \mathrm{~m}$ of the sensor array perimeter 
Table 1 Parameter values used in the simulations

\begin{tabular}{ll}
\hline Parameter & Value \\
\hline Number of sensors $(N)$ & 20 \\
Locations of sensors & Grid formation with separations of $50 \mathrm{~m}$ (see Fig. 1) \\
Probability of detection of each target $\left(P_{d}\right)$ & $0.8,0.9$, or 1.0 \\
Average false alarms per sensor & 2.2 \\
AOA measurement error standard deviation $\left(\sigma_{\theta}\right)$ & $1^{0}$ \\
DDOA measurement error standard deviation $\left(\sqrt{2} c \sigma_{t}\right)$ & $10 \mathrm{~m}$ \\
Signal propagation speed, $c$ & $343.2 \mathrm{~m} / \mathrm{s}$ (i.e. the speed of sound in air) \\
Number of target events & Up to 10 \\
Target event locations & Uniformly sampled in the surveillance region $[3 \mathrm{~km}, 7 \mathrm{~km}] \times[-2 \mathrm{~km}, 2 \mathrm{~km}]$, but excluding \\
Target event times & events from occurring within $500 \mathrm{~m}$ of the sensor array (again, see Fig. 3) \\
\hline
\end{tabular}

where $e_{\theta}($.$) and e_{t}($.$) , and zero-mean, Gaussian distributed$ measurement errors with standard deviations $\sigma_{\theta}$ and $\sigma_{t}$ respectively; $c$ is the signal propagation speed; and $d\left(X_{k}\right)$ is the distance between the target and the sensor, i.e.:

$$
d\left(\boldsymbol{X}_{k}\right)=\sqrt{\left(x_{k}-x_{S}\right)^{2}+\left(y_{k}-y_{S}\right)^{2}}
$$

where $\left(x_{S}, y_{S}\right)$ are the Cartesian coordinates of the sensor.

\subsubsection{DDOA measurement generation}

Typically, the times $t_{k}$ at which the target events occur are unknown. However, these times can be factored out of the analysis by calculating the difference between the TOA measurements generated by different sensors (assuming the signal originates from the same target), giving measurements of the time difference of arrival (TDOA). Multiplying the TDOA by the signal propagation speed, and under the assumption that the two TOA measurements are generated by the same target event, the distance difference of arrival (DDOA) between TOA measurements generated by sensors $i$ and $j$ is given as follows:

$$
\begin{aligned}
\tau(i, j) & \triangleq c[t(i)-t(j)] \\
& =\left[d_{i}\left(\boldsymbol{X}_{k}\right)-d_{j}\left(\boldsymbol{X}_{k}\right)\right]+c\left[e_{t}(i)-e_{t}(j)\right]
\end{aligned}
$$

Importantly, Eq. (31) is independent of $t_{k}$. The exploitation of TDOA measurements has been an area of great interest for over three decades [33]. Each TDOA measurement provides a hyperbola of potential target locations, and the intersection of two such hyperbola enables the target location to be estimated (e.g. see [34]).

In determining the DDOA measurements, it is important to be aware of the potential for data incest (i.e. using the same information more than once), especially when one considers the following:

$$
\tau(i, k)=\tau(i, j)+\tau(j, k)
$$

In order to determine a unique set of DDOAs, in which no measurement is a linear combination of any of the other measurements, the DDOAs are calculated between a reference sensor (denoted throughout by the index " $R$ ") and each of the other sensors. It is noted that the DDOA measurements generated by different sensors are correlated, because they utilise the same TOA measurement generated by the reference sensor. Indeed, it can easily be shown that $\operatorname{Cov}[\tau(R, i), \tau(R, j)]=c^{2} \sigma_{t}^{2}$ for $i \neq j$; and $\operatorname{Cov}[\tau(R, i), \tau(R, i)]=2 c^{2} \sigma_{t}^{2}$ for $i=1, \ldots, N$.

The reference sensor is chosen to be the sensor at which the greatest number of measurements are generated. This ensures that the largest possible set of potential DDOA measurements is created. This is particularly important if there are missed detections, because in such cases a poor choice of reference sensor (i.e. choosing one at which very few measurements are generated) could severely restrict the number of DDOA measurement combinations evaluated, which might negatively impact on the ability of the TALA to subsequently detect and localise the target events.

Again, let $n(i)$ denote the number of measurements generated by sensor $i$, which can include both target generated measurements and spurious false alarms. The total number of DDOA measurement combinations is then $\left[n(R) \times \sum_{i \neq R} n(i)\right]$. However, it follows immediately 
from the reverse triangle inequality that:

$$
\left|d_{R}\left(\boldsymbol{X}_{k}\right)-d_{i}\left(\boldsymbol{X}_{k}\right)\right| \leq \sqrt{\left(x_{S_{R}}-x_{S_{i}}\right)^{2}+\left(y_{S_{R}}-y_{S_{i}}\right)^{2}} \text { for all } i
$$

The inequality (33) states that the magnitude of each ground-truth DDOA measurement can be no greater than the distance between the reference sensor and sensor $i$. Motivated by this, DDOA measurements are only considered if they satisfy the following inequality:

$$
|\tau(R, i)| \leq \sqrt{\left(x_{S_{R}}-x_{S_{i}}\right)^{2}+\left(y_{S_{R}}-y_{S_{i}}\right)^{2}}+\gamma \sigma_{\tau}
$$

where $\sigma_{\tau} \triangleq \sqrt{2} c \sigma_{t}$ is the DDOA measurement error standard deviation; and $\gamma \geq 0$ is a pre-specified multiplier. If $\gamma \gg 1$, DDOA measurements that do not satisfy Eq. (34) are likely to have been calculated from TOA measurements generated by different targets, although Eq. (34) can also be violated if TOA measurement errors are abnormally large.

\subsection{TALA implementation}

The TALA implementations in the three scenarios have the following features:

1. In scenarios $1-2$, the TALA is implemented using the coupled AOA/DDOA measurement pairs, with the reference sensor providing only AOA measurements.

2. It is noted that the DDOA measurements determined for different sensors are correlated, because they exploit the same TOA measurement set generated by the reference sensor. The correlation between between the DDOA measurements at different sensors is $c^{2} \sigma_{t}^{2}$. As a result, the matrix manipulations within the TALA cannot exploit the redundancy created had the measurement error covariance matrix $\boldsymbol{\Sigma}$ been (block) diagonal ${ }^{5}$.

3. In scenarios 1 and 3 , initial candidate locations are generated as the intersection of each pair of AOA measurements and the intersection of each pair of DDOA measurements. Calculating the AOA intersections is straightforward. However, calculating the DDOA intersections is more problematic, and an iterative $\mathrm{N}-\mathrm{R}$ approach is used. Full details are given in Appendix A.

4. Scenario 2 is the most computationally complex because, respectively, the number of DDOA combinations that satisfy Eq. (34) is greater than in scenario 1 , and the dimensionality of the concatenated measurement vector is greater than in scenario 3 . As a result, for scenario 2:

- Only the AOA intersections are used to generate initial candidate locations.
- The correlations between the DDOA measurements are ignored (which simplifies the matrix manipulations within the TALA, e.g. see endnote 5).

5. In all three scenarios, in order to reduce the computational complexity of the TALA, the initial candidate locations are determined only from the intersections of measurements generated by five of the sensors.

6. In all three scenarios, deletion criterion 3 is used on Step 3. Therefore, the intersection deletion step only allows each AOA measurement to be exploited by one candidate location. As a result, in scenarios 1-2, the intersection deletion step must account for the fact that some AOA/DDOA measurement pairs share a common AOA measurement.

7. Only in scenario 3 is measurement reassociation performed during the G-N gradient descent.

A summary of the TALA settings is provided in Table 2. It is noted that some elements of the implementations (e.g. ignoring measurement correlations) can compromise algorithm performance for increased computational speed.

\subsection{Performance evaluation}

Results are based on 1000 simulations of each scenario; with either $1,2, \ldots$, or 10 target events, and $P_{d}$ values of $0.8,0.9$, and 1.0. In comparing the performance of the TALA with that of the gTALA and the CRLB, the overall location RMSE is calculated in each case. For the TALA, the overall location RMSE is given as follows:

overall location RMSE $=\sqrt{\frac{1}{N_{E}} \sum_{i=1}^{N_{E}}\left[M S E_{i}(x)+M S E_{i}(y)\right]}$

where:

$$
\begin{aligned}
M S E_{i}(x) \triangleq & \text { MSE of estimate } i \text { in the } \\
& x \text {-coordinate direction } \\
M S E_{i}(y) \triangleq & \text { MSE of estimate } i \text { in the } \\
& y \text {-coordinate direction } \\
N_{E} \triangleq & \text { total number of target events } \\
& \text { detected (across the } 1000 \text { simulations) }
\end{aligned}
$$

In determining the MSE values, a global nearest neighbour approach is used to pair the estimates with the true target event locations. An estimate is declared to be a successful detection if the absolute distance error of the estimate is less than 10 times the CRLB location RMSE for that event, otherwise the estimate is declared to be a false event. 
Table 2 Summary of TALA settings

\begin{tabular}{|c|c|}
\hline TALA component & Details \\
\hline AOA/DDOA intersections (Step 1) & $\begin{array}{l}\text { Based on measurements at only five sensors, only DDOAs satisfying Eq. (34) are used, } \\
\text { with } \gamma=2\end{array}$ \\
\hline N-R approach to determine DDOA intersections (Step 1) & $\begin{array}{l}10 \text { attempts allowed: attempt \#1 initialises with the corresponding AOA intersection, } \\
\text { subsequent attempts use random starting locations }\end{array}$ \\
\hline Measurement association (Step 2) & Gate threshold, $\xi=10^{-3}$ (scenarios 1-2) gate threshold, $\xi=10^{-2}$ (scenarios 3) \\
\hline Intersection downselection (Step 3) & $\begin{array}{l}\text { Only consider intersections for which the total number of associated measurements } \\
\text { is no smaller than } N P_{d} / 2\end{array}$ \\
\hline \multirow[t]{4}{*}{ G-N approach for ML estimation (Step 4) } & $\begin{array}{l}\text { Determine overall likelihood for step sizes of } \pm 20 \%, \pm 40 \%, \pm 60 \%, \pm 80 \% \text {, and } \pm 100 \% \\
\text { of the Newton step (i.e. } m=5 \text { ) }\end{array}$ \\
\hline & $\begin{array}{l}\text { If no step increases the overall likelihood, consider a step of magnitude } 200 \mathrm{~m} \text { (i.e. } \\
\left.=\delta_{M}\right) \text { in a random direction }\end{array}$ \\
\hline & $\begin{array}{l}\text { Stop iterating if the procedure above has attempted a total of } 20 \text { random steps, or } \\
\text { each component of the GNSSE } \boldsymbol{F}\left(X_{k}\right)^{\prime} \boldsymbol{\Sigma}^{-1} \times\left[\boldsymbol{Z}-\boldsymbol{f}\left(X_{k}\right)\right] \text { has a magnitude smaller } \\
\text { than } 10^{-3}\end{array}$ \\
\hline & Reassociation is only performed during gradient descent in scenario 3 \\
\hline
\end{tabular}

The gTALA and CRLB overall location RMSEs are calculated in similar fashion, but do not require the pairing step because, in each case, the target event is pre-specified in each RMSE calculation. In order to reduce the impact of outliers, in Tables 3, 5, and 7, the overall location RMSEs are also calculated when the $1 \%$ worst geo-location estimates (in terms of the TALA overall location RMSE) are excluded from each calculation.

The percentage of target events detected (\%E) and the average number of false events declared (\#FE) are then given as follows:

$$
\begin{aligned}
\% \mathrm{E} & =100 \times N_{E} / T_{E} \\
\# \mathrm{FE} & =N_{F E} / 1000
\end{aligned}
$$

where $T_{E}$ is the total number of events across the 1000 simulations, and $N_{F E}$ is the total number of false events declared across the 1000 simulations.

\subsection{Simulation results}

Simulation results are presented in Figs. 4, 5 and 6 and Tables 3, 4, 5, 6, 7 and 8. All simulations were run on an Intel Core $^{\mathrm{TM}} \mathrm{i} 5-430 \mathrm{M}$ processor $(2.26 \mathrm{GHz})$.

Firstly, in Fig. 4 and Table 3, the estimation accuracy of the TALA is shown for scenario 1. Estimation performance is similar to both the gTALA performance and the optimistic CRLB. Indeed, the RMSEs of the TALA and gTALA typically differ by less than $10 \%$, indicating that the TALA is extremely good and making the correct associations of measurements to target events. In fact, the TALA makes the correct measurement-to-target association over $93 \%$ of the time in this scenario. Furthermore, the RMSE of the TALA is typically within $10-20 \%$ of the CRLB. When outliers are removed from the analysis, differences in performance are even smaller, with the TALA RMSE typically around 5\% greater than both the gTALA RMSE and the CRLB. The TALA is able to identify and geo-locate $93-98 \%$ of the target events (see column 1 in Table 3). Moreover, the average number of false events declared (i.e. estimates that are too geographically distant from a true target event to be considered to be detection/localisation of an event) is negligible (see column 2 in Table 3).

Table 4 shows the average computational time of the TALA for scenario 1 . It is observed that for single events, the TALA provides almost instantaneous estimates. As the number of target events increases, the algorithm runtime increases exponentially, primarily because the number of AOA and DDOA intersections that have to be computed and manipulated increases exponentially (see column 2). Nevertheless, the algorithm is still able to provide estimates of the geo-locations of up to 10 target events in around $1 \mathrm{~s}$.

In Fig. 5 and Tables 5 and 6, the performance of the TALA is shown for scenario 2, the first of the scenarios with simultaneous target events. In this case, the number of DDOA combinations that satisfy Eq. (34) is increased. Therefore, the measurement-to-target association problem has greater complexity, which significantly increases the complexity/run-time of the TALA. In this case, in order to reduce the complexity of the TALA, only the AOA intersections are used to generate initial candidate locations, and the TALA ignores the correlations between the DDOA measurements. The following observations are made. 
Table 3 Summary of the TALA geo-location performance for scenario 1

\begin{tabular}{|c|c|c|c|c|c|c|c|c|c|}
\hline \multicolumn{5}{|c|}{ TALA (0\% exclusions) } & \multicolumn{3}{|c|}{ TALA (1\% exclusions) } & \multirow{2}{*}{ Events } & \multirow{2}{*}{$P_{d}$} \\
\hline$\% \overline{\mathrm{E}}$ & \#FE & RMSE & $\% \uparrow$ gTALA & $\% \uparrow C R L B$ & RMSE & $\% \uparrow$ gTALA & $\% \uparrow C R L B$ & & \\
\hline 98.10 & 0.00 & 216.98 & 2.80 & 7.72 & 195.97 & 2.36 & -0.78 & 1 & 1.0 \\
\hline 98.40 & 0.00 & 238.12 & 2.72 & 14.92 & 206.67 & 2.76 & 1.72 & 2 & 1.0 \\
\hline 97.77 & 0.00 & 232.70 & 3.01 & 13.87 & 197.37 & 3.31 & -1.54 & 3 & 1.0 \\
\hline 98.13 & 0.00 & 239.33 & 6.25 & 18.18 & 214.38 & 5.95 & 7.97 & 4 & 1.0 \\
\hline 98.06 & 0.00 & 227.99 & 5.50 & 12.77 & 200.64 & 4.65 & 1.25 & 5 & 1.0 \\
\hline 97.95 & 0.00 & 226.04 & 5.20 & 10.06 & 198.73 & 4.53 & -1.34 & 6 & 1.0 \\
\hline 98.17 & 0.00 & 231.29 & 5.48 & 13.60 & 203.29 & 5.17 & 1.83 & 7 & 1.0 \\
\hline 97.99 & 0.00 & 234.62 & 3.84 & 14.51 & 201.89 & 4.46 & 0.46 & 8 & 1.0 \\
\hline 98.12 & 0.00 & 231.39 & 6.28 & 13.25 & 202.37 & 5.91 & 0.97 & 9 & 1.0 \\
\hline 98.14 & 0.01 & 230.68 & 5.62 & 13.69 & 198.47 & 5.60 & -0.19 & 10 & 1.0 \\
\hline 96.80 & 0.00 & 256.88 & 7.90 & 19.27 & 220.50 & 7.01 & 4.92 & 1 & 0.9 \\
\hline 97.20 & 0.00 & 247.17 & 4.10 & 12.19 & 220.18 & 6.73 & 2.10 & 2 & 0.9 \\
\hline 97.23 & 0.00 & 248.38 & 5.54 & 14.06 & 215.66 & 5.39 & 1.35 & 3 & 0.9 \\
\hline 96.53 & 0.00 & 255.22 & 4.76 & 18.13 & 222.20 & 4.94 & 5.06 & 4 & 0.9 \\
\hline 96.84 & 0.00 & 263.92 & 9.34 & 22.73 & 222.88 & 6.10 & 5.89 & 5 & 0.9 \\
\hline 97.28 & 0.00 & 255.65 & 6.46 & 16.71 & 222.66 & 6.85 & 3.83 & 6 & 0.9 \\
\hline 96.80 & 0.00 & 261.96 & 6.25 & 20.74 & 223.58 & 6.88 & 5.28 & 7 & 0.9 \\
\hline 96.78 & 0.01 & 256.22 & 8.48 & 17.31 & 223.52 & 8.00 & 4.59 & 8 & 0.9 \\
\hline 97.14 & 0.00 & 247.81 & 5.32 & 13.90 & 221.65 & 5.83 & 4.09 & 9 & 0.9 \\
\hline 96.91 & 0.01 & 250.97 & 7.88 & 16.14 & 217.35 & 7.57 & 2.86 & 10 & 0.9 \\
\hline 94.30 & 0.00 & 251.86 & 6.68 & 9.02 & 231.15 & 7.78 & 2.70 & 1 & 0.8 \\
\hline 94.65 & 0.00 & 278.05 & 6.17 & 17.02 & 236.68 & 5.41 & 1.91 & 2 & 0.8 \\
\hline 94.97 & 0.00 & 278.78 & 5.58 & 18.50 & 241.75 & 7.93 & 5.44 & 3 & 0.8 \\
\hline 94.90 & 0.00 & 281.74 & 8.78 & 21.92 & 240.55 & 6.93 & 6.52 & 4 & 0.8 \\
\hline 95.14 & 0.01 & 279.13 & 6.49 & 20.70 & 240.08 & 6.44 & 6.26 & 5 & 0.8 \\
\hline 95.22 & 0.01 & 273.00 & 4.20 & 16.35 & 234.65 & 5.16 & 2.24 & 6 & 0.8 \\
\hline 94.76 & 0.01 & 291.93 & 10.05 & 25.12 & 239.95 & 7.45 & 5.25 & 7 & 0.8 \\
\hline 94.46 & 0.01 & 281.12 & 6.25 & 19.67 & 236.85 & 7.05 & 3.26 & 8 & 0.8 \\
\hline 93.99 & 0.01 & 285.59 & 7.74 & 22.04 & 236.52 & 6.47 & 3.60 & 9 & 0.8 \\
\hline 93.47 & 0.02 & 280.47 & 7.25 & 20.47 & 237.02 & 7.32 & 4.36 & 10 & 0.8 \\
\hline
\end{tabular}

Shown are the percentage of target events detected (\%E), the average number of false events declared (\#FE), and the geolocation RMSEs (in metres). RMSEs are shown for all target geo-locations determined across the 1000 simulations (i.e. 0\% exclusions), and with the worst $1 \%$ of geo-location estimates excluded from each RMSE calculation. Also shown are the percentage increase of the TALA RMSE over the gTALA RMSE (\% $\uparrow$ gTALA) and the percentage increase of the TALA RMSE over the CRLB RMSE (\%个 CRLB)

1. The geo-location performance of the TALA again compares well with the gTALA and the CRLB (see Fig. 5 and Table 5), with a degradation in geo-location performance of just a few percent compared to scenario 1 .

2. The TALA again detects $93-98 \%$ of the target events (see column 1 of Table 5).

3. The average number of false events declared is again negligible (see column 2 of Table 5).
4. The additional complexity of the data association problem has been negated by the changes to the TALA. As a result, in the most complex cases (i.e. with 10 target events), the TALA run-time remains less than $2 \mathrm{~s}$ (see Table 6).

In Fig. 6 and Tables 7 and 8, the performance of the TALA is shown for scenario 3 . This is the most difficult scenario, because in addition to target events 

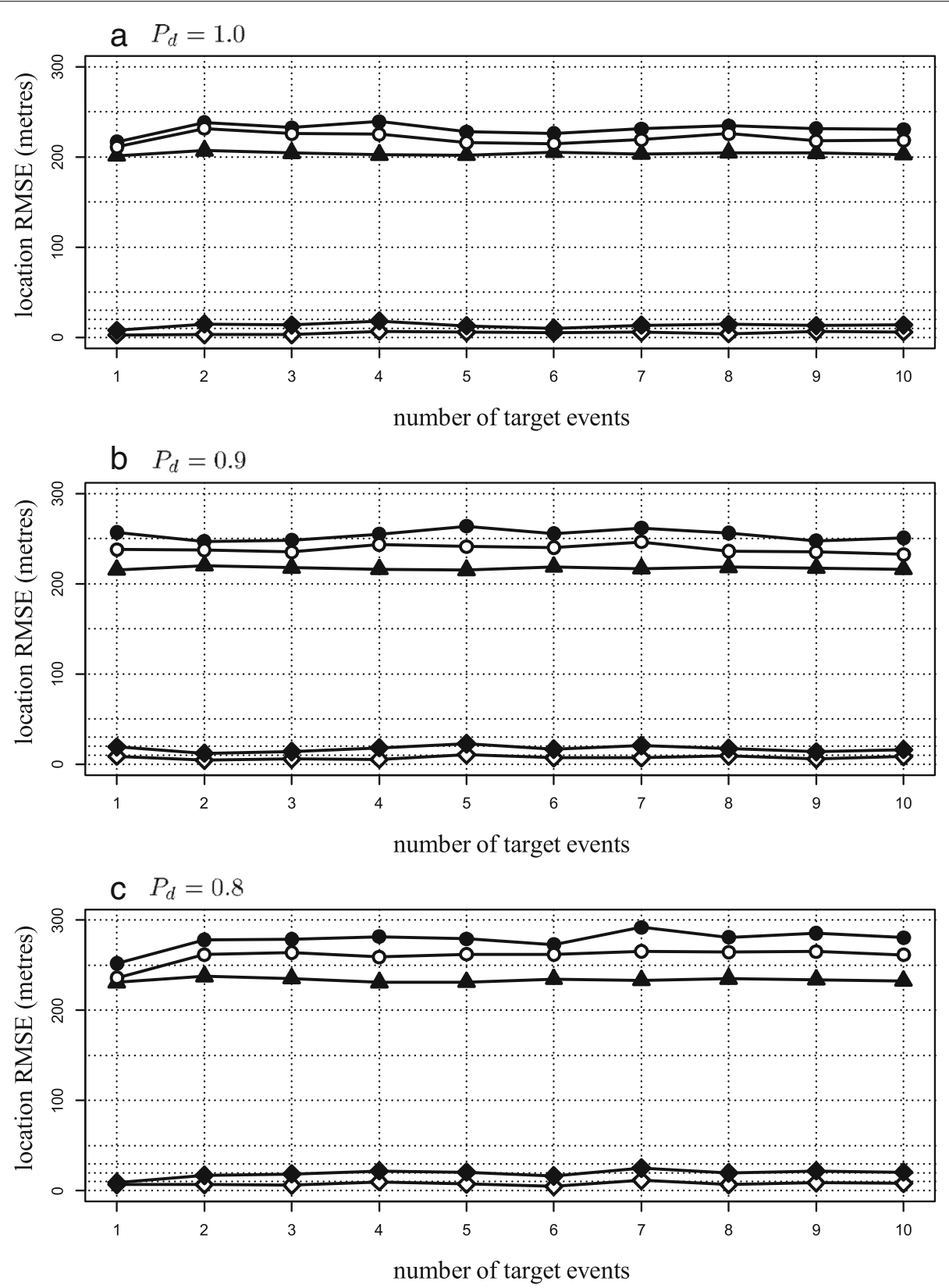

Fig. 4 Target localisation performance for scenario 1. The TALA RMSE (black circles), gTALA RMSE (white circles), and the CRLB RMSE (black triangles). The percentage differences between the performance of the TALA and (i) the CRLB (black diamonds) and (ii) the gTALA (white diamonds). Results are averaged over all 1000 simulations. a $P_{d}=1.0$. b $P_{d}=0.9$. $\mathbf{c} P_{d}=0.8$

occurring simultaneously, each sensor provides only AOA or TOA measurements, and not hybrid AOA/TOA measurements. This makes measurement association more problematic. For this reason, measurement reassociation is performed on each iteration of the gradient descent algorithm, following the procedure described in Section 2.6.3. However, the reduced size of the concatenated measurement vector, as a result of the reduction in the dimensionality of each measurement, does not necessitate the computational adjustments to the TALA that were required for scenario 2 .

In scenario 3 , the geo-location performance of the TALA again compares well with the gTALA and the CRLB (see Fig. 6 and Table 7), although indicative of the more difficult scenario, percentage differences in performance are greater than previously. To elaborate, the RMSEs of the TALA and gTALA typically differ by $10-20 \%$, with the RMSE of the TALA typically $20-50 \%$ greater than the 

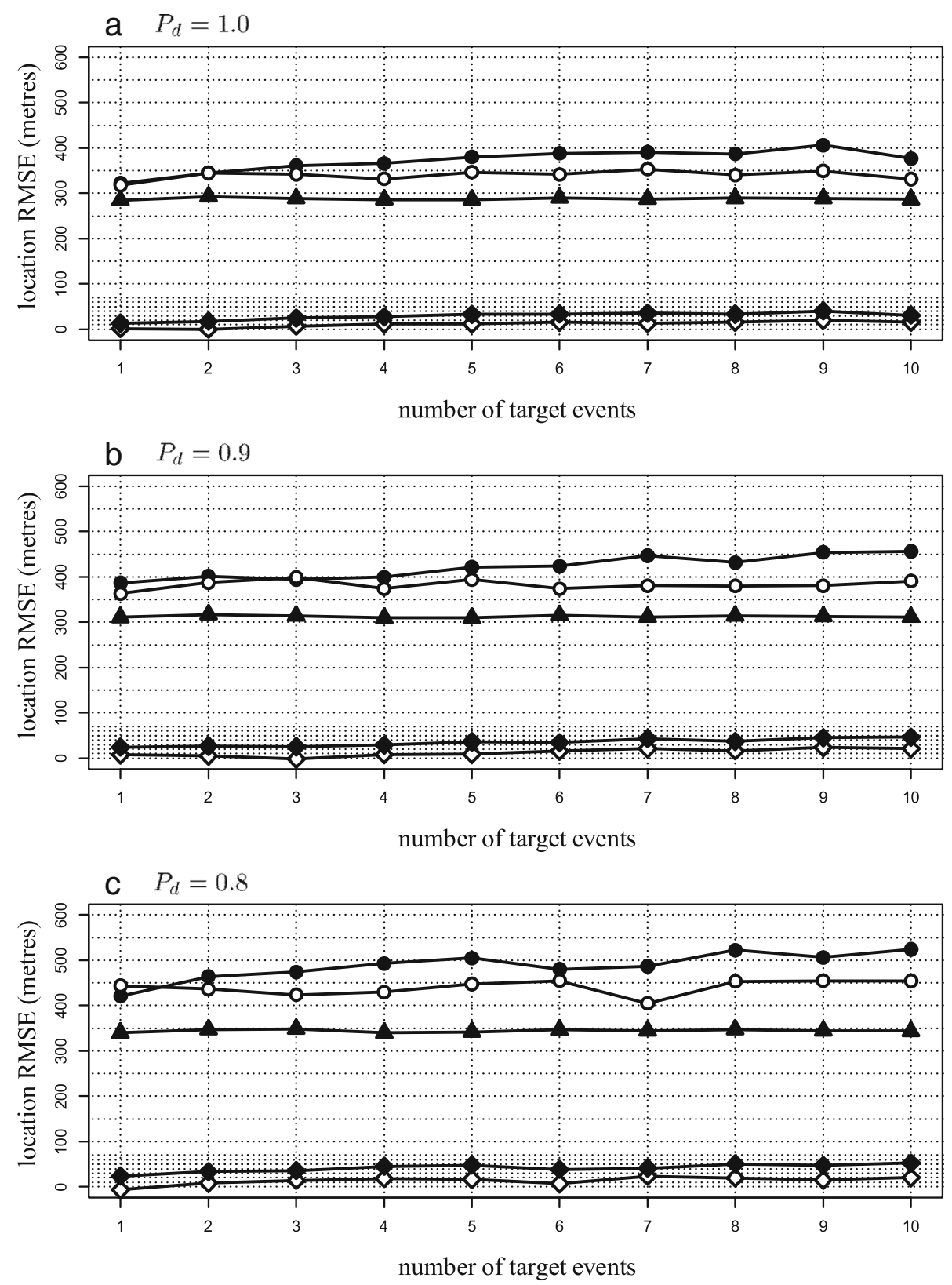

Fig. 5 Target localisation performance for scenario 2. The TALA RMSE (black circles), gTALA RMSE (white circles), and the CRLB RMSE (black triangles). The percentage differences between the performance of the TALA and (i) the CRLB (black diamonds) and (ii) the gTALA (white diamonds). Results are averaged over all 1000 simulations. a $P_{d}=1.0 . \mathbf{b} P_{d}=0.9$. $\mathbf{c} P_{d}=0.8$

CRLB. When outliers are removed from the analysis, differences in performance are significantly reduced, with the TALA RMSE typically within $20 \%$ of both the gTALA RMSE and the CRLB. The TALA detects $83-99 \%$ of the target events (see column 1 of Table 7), whilst the average number of false events remains extremely low (see column 2 of Table 7). The reduced computational complexity of the algorithm, as a result of the reduction in the dimensionality of each concatenated measurement vector, enables the TALA to generate estimates in the most complex scenarios in less than $0.5 \mathrm{~s}$ (see Table 8).

In all three scenarios, the performance of the TALA was sensitive to the gate threshold $\xi$. Setting the value of $\xi$ too high can increase the proportion of incorrect associations. This is particularly true in scenarios for which $P_{d}<1$, in which case there is an increased likelihood that a false measurement will be associated with a candidate location whenever a sensor fails to make a target detection. 

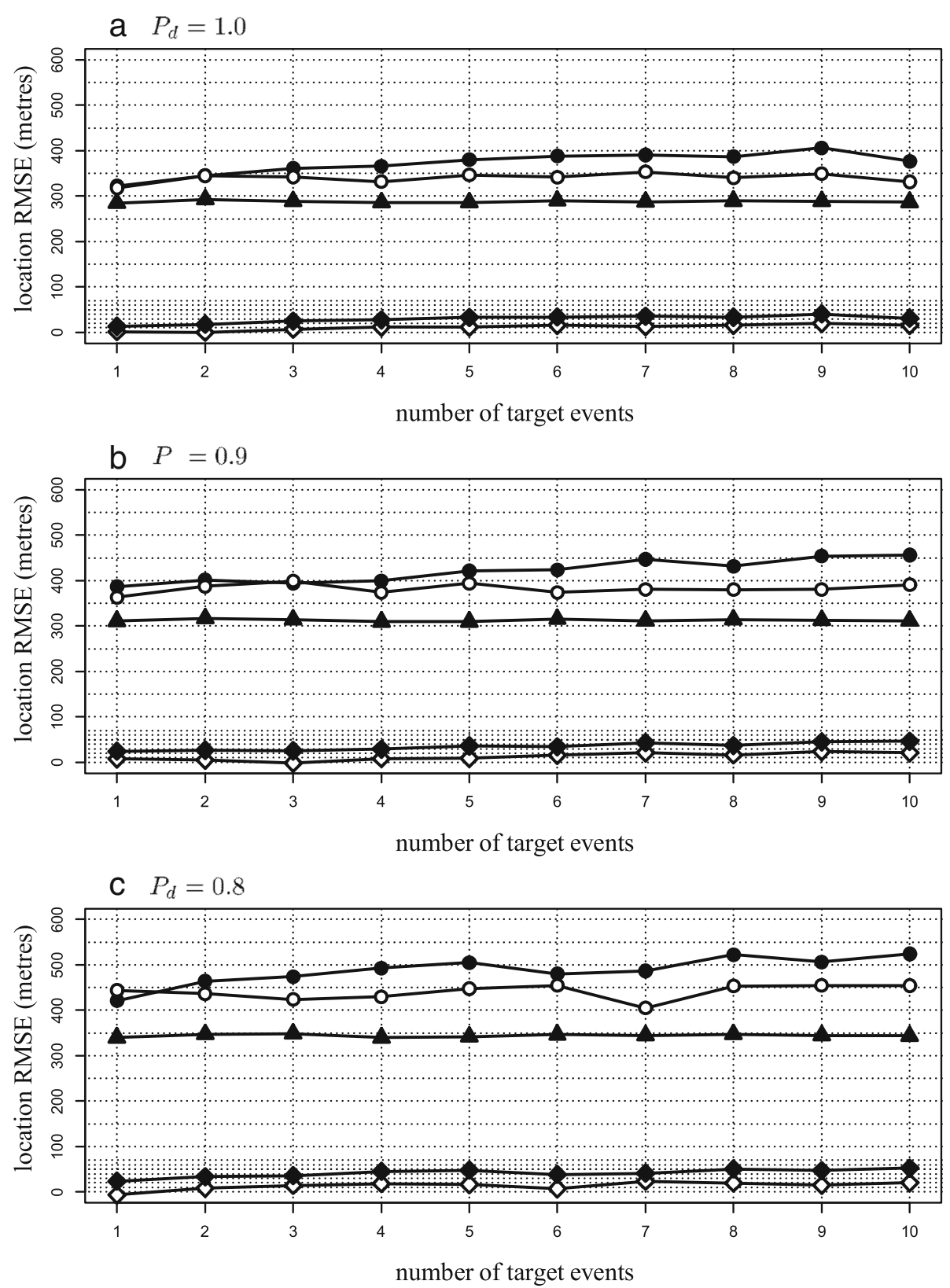

Fig. 6 Target localisation performance for scenario 3. The TALA RMSE (black circles), gTALA RMSE (white circles), and the CRLB RMSE (black triangles) The percentage differences between the performance of the TALA and (i) the CRLB (black diamonds) and (ii) the gTALA (white diamonds). Results are averaged over all 1000 simulations. a $P_{d}=1.0 . \mathbf{b} P_{d}=0.9$. $\mathbf{c} P_{d}=0.8$

Conversely, setting the value of $\xi$ too low can result in target-generated measurements failing to be gated. Via extensive experimentation in all three scenarios, threshold values in the range $10^{-3}-10^{-2}$ were shown to result in near optimal performance. This is equivalent to gating the measurement Mahalanobis distance with a threshold $g$ in the range 3.0-3.7.

The performance of the TALA was also assessed for both closely spaced and well separated emitters, and the results are similar to those presented herein, with only a few percent difference in the resulting RMSE, \%E, and \#FE values. Additionally, a Newton-Raphson (N-R) approach (e.g. [25]) was implemented in order to perform the gradient descent within the TALA. The N-R approach had similar performance to the G-N approach, with the exception that the computational expense of the N-R algorithm was greater, as a result of the requirement to calculate second-order derivative terms. 
Table 4 TALA average computational time, and the average number of candidate locations at the end of each step of the TALA, for scenario 1

\begin{tabular}{|c|c|c|c|c|c|c|c|c|}
\hline \multirow{3}{*}{$\begin{array}{l}\text { TALA } \\
\text { run time } \\
\text { (s) }\end{array}$} & \multicolumn{6}{|c|}{ Average number of candidate positions } & \multirow{3}{*}{ Events } & \multirow{3}{*}{$P_{d}$} \\
\hline & Step 1 & Step 3 & Step 3 & Step 3 & Step 4 & Step 5 & & \\
\hline & & (crit 1) & (crit 2) & (crit 3) & & & & \\
\hline 0.01 & 20.01 & 8.63 & 4.35 & 0.98 & 0.98 & 0.98 & 1 & 1.0 \\
\hline 0.04 & 50.19 & 18.31 & 9.07 & 1.97 & 1.97 & 1.97 & 2 & 1.0 \\
\hline 0.05 & 90.41 & 28.47 & 14.16 & 2.94 & 2.94 & 2.93 & 3 & 1.0 \\
\hline 0.11 & 146.74 & 39.78 & 19.62 & 3.94 & 3.94 & 3.93 & 4 & 1.0 \\
\hline 0.19 & 217.19 & 53.06 & 25.81 & 4.92 & 4.92 & 4.90 & 5 & 1.0 \\
\hline 0.29 & 295.72 & 67.31 & 32.21 & 5.90 & 5.90 & 5.88 & 6 & 1.0 \\
\hline 0.41 & 392.83 & 79.28 & 38.40 & 6.90 & 6.90 & 6.87 & 7 & 1.0 \\
\hline 0.61 & 489.83 & 95.09 & 45.99 & 7.87 & 7.87 & 7.84 & 8 & 1.0 \\
\hline 0.77 & 613.30 & 109.80 & 52.78 & 8.87 & 8.87 & 8.84 & 9 & 1.0 \\
\hline 1.03 & 747.38 & 127.43 & 61.14 & 9.85 & 9.85 & 9.82 & 10 & 1.0 \\
\hline 0.02 & 17.09 & 7.04 & 3.53 & 0.97 & 0.97 & 0.97 & 1 & 0.9 \\
\hline 0.02 & 41.22 & 14.80 & 7.50 & 1.95 & 1.95 & 1.94 & 2 & 0.9 \\
\hline 0.07 & 76.86 & 23.16 & 11.84 & 2.92 & 2.92 & 2.92 & 3 & 0.9 \\
\hline 0.13 & 120.39 & 32.24 & 16.36 & 3.88 & 3.88 & 3.86 & 4 & 0.9 \\
\hline 0.18 & 177.15 & 42.50 & 21.23 & 4.87 & 4.87 & 4.84 & 5 & 0.9 \\
\hline 0.23 & 242.85 & 54.03 & 26.81 & 5.86 & 5.86 & 5.84 & 6 & 0.9 \\
\hline 0.35 & 319.59 & 63.91 & 31.86 & 6.81 & 6.81 & 6.78 & 7 & 0.9 \\
\hline 0.47 & 393.38 & 76.07 & 37.77 & 7.78 & 7.78 & 7.75 & 8 & 0.9 \\
\hline 0.60 & 494.43 & 88.45 & 43.77 & 8.77 & 8.77 & 8.75 & 9 & 0.9 \\
\hline 0.86 & 606.16 & 102.99 & 50.96 & 9.74 & 9.74 & 9.70 & 10 & 0.9 \\
\hline 0.02 & 15.21 & 5.65 & 2.96 & 0.95 & 0.95 & 0.94 & 1 & 0.8 \\
\hline 0.03 & 34.04 & 11.86 & 6.13 & 1.90 & 1.90 & 1.90 & 2 & 0.8 \\
\hline 0.04 & 60.84 & 18.28 & 9.58 & 2.86 & 2.86 & 2.85 & 3 & 0.8 \\
\hline 0.10 & 98.99 & 25.73 & 13.55 & 3.81 & 3.81 & 3.80 & 4 & 0.8 \\
\hline 0.16 & 144.13 & 34.58 & 18.10 & 4.78 & 4.78 & 4.76 & 5 & 0.8 \\
\hline 0.22 & 195.82 & 44.28 & 22.70 & 5.74 & 5.74 & 5.72 & 6 & 0.8 \\
\hline 0.29 & 254.63 & 51.31 & 26.48 & 6.68 & 6.68 & 6.64 & 7 & 0.8 \\
\hline 0.40 & 320.04 & 61.82 & 31.65 & 7.61 & 7.61 & 7.57 & 8 & 0.8 \\
\hline 0.50 & 393.89 & 71.18 & 36.43 & 8.50 & 8.50 & 8.47 & 9 & 0.8 \\
\hline 0.67 & 470.19 & 81.29 & 41.61 & 9.39 & 9.39 & 9.36 & 10 & 0.8 \\
\hline
\end{tabular}

\section{Discussion}

There is a tradeoff between the run-time and the performance of the TALA. Adjustments that reduce the run-time, such as reducing the initial number of candidate locations (e.g. by not considering all measurement intersections), ignoring the correlations between DDOA measurements (e.g. to simplify the matrix manipulations in the gradient descent), and more quickly reducing the number of candidate locations (e.g. by performing downselection on Step 3), can each reduce the proportion of emitters detected, although performance degradation is not significant. Furthermore, performing measurement reassociation during gradient descent can improve the TALA performance, particularly if measurement errors are large or the sensors provide only limited information (e.g. AOA measurements only), rather than hybrid measurements. However, measurement reassociation also increases the computational expense of the TALA. 
Table 5 Summary of the TALA geo-location performance for scenario 2

\begin{tabular}{|c|c|c|c|c|c|c|c|c|c|}
\hline \multicolumn{5}{|c|}{ TALA (0\% exclusions) } & \multicolumn{3}{|c|}{ TALA (1\% exclusions) } & \multirow{2}{*}{ Events } & \multirow{2}{*}{$P_{d}$} \\
\hline$\% \overline{\mathrm{E}}$ & $\# F E$ & RMSE & $\% \uparrow$ gTALA & $\% \uparrow C R L B$ & RMSE & $\% \uparrow$ gTALA & $\% \uparrow C R L B$ & & \\
\hline 98.20 & 0.00 & 221.55 & 4.97 & 9.99 & 199.36 & 4.13 & 0.93 & 1 & 1.0 \\
\hline 98.40 & 0.00 & 240.13 & 3.59 & 15.89 & 208.14 & 3.49 & 2.44 & 2 & 1. \\
\hline 97.67 & 0.00 & 235.85 & 4.40 & 15.41 & 198.36 & 3.83 & -1.05 & 3 & 1.0 \\
\hline 98.02 & 0.00 & 240.26 & 6.67 & 18.65 & 214.88 & 6.20 & 8.23 & 4 & 1. \\
\hline 97.72 & 0.00 & 227.59 & 5.31 & 12.57 & 203.19 & 5.98 & 2.54 & 5 & 1. \\
\hline 97.73 & 0.00 & 230.52 & 7.29 & 12.24 & 202.06 & 6.28 & 0.32 & 6 & 1.0 \\
\hline 97.86 & 0.00 & 233.17 & 6.34 & 14.53 & 204.34 & 5.71 & 2.35 & 7 & 1. \\
\hline 97.75 & 0.00 & 236.42 & 4.63 & 15.39 & 203.31 & 5.19 & 1.17 & 8 & 1. \\
\hline 97.83 & 0.00 & 235.20 & 8.03 & 15.11 & 204.10 & 6.82 & 1.83 & 9 & 1. \\
\hline 97.76 & 0.00 & 235.39 & 7.78 & 16.02 & 202.92 & 7.96 & 2.04 & 10 & 1.0 \\
\hline 96.60 & 0.00 & 261.04 & 9.65 & 21.20 & 222.55 & 8.01 & 5.89 & 1 & 0.9 \\
\hline 97.20 & 0.00 & 248.90 & 4.82 & 12.97 & 221.57 & 7.40 & 2.75 & 2 & 0.9 \\
\hline 97.00 & 0.00 & 250.68 & 6.52 & 15.11 & 219.06 & 7.06 & 2.95 & 3 & 0.9 \\
\hline 96.60 & 0.00 & 260.54 & 6.95 & 20.60 & 227.29 & 7.34 & 7.47 & 4 & 0.9 \\
\hline 96.50 & 0.01 & 262.33 & 8.68 & 21.99 & 225.08 & 7.15 & 6.94 & 5 & 0.9 \\
\hline 97.08 & 0.00 & 259.16 & 7.92 & 18.31 & 224.81 & 7.88 & 4.84 & 6 & 0.9 \\
\hline 96.49 & 0.00 & 264.01 & 7.08 & 21.68 & 226.14 & 8.10 & 6.49 & 7 & 0.9 \\
\hline 96.38 & 0.01 & 262.57 & 11.17 & 20.22 & 227.18 & 9.76 & 6.30 & 8 & 0.9 \\
\hline 96.66 & 0.01 & 253.79 & 7.86 & 16.65 & 226.42 & 8.10 & 6.33 & 9 & 0.9 \\
\hline 96.25 & 0.01 & 257.98 & 10.90 & 19.39 & 221.94 & 9.84 & 5.04 & 10 & 0.9 \\
\hline 94.30 & 0.00 & 251.48 & 6.52 & 8.85 & 232.14 & 8.24 & 3.14 & 1 & 0.8 \\
\hline 94.45 & 0.01 & 284.93 & 8.80 & 19.91 & 238.50 & 6.22 & 2.69 & 2 & 0.8 \\
\hline 95.00 & 0.00 & 285.47 & 8.11 & 21.34 & 246.61 & 10.10 & 7.56 & 3 & 0.8 \\
\hline 94.55 & 0.00 & 287.55 & 11.02 & 24.43 & 244.97 & 8.90 & 8.48 & 4 & 0.8 \\
\hline 94.74 & 0.01 & 289.19 & 10.32 & 25.05 & 239.86 & 6.35 & 6.17 & 5 & 0.8 \\
\hline 94.87 & 0.01 & 277.23 & 5.81 & 18.15 & 238.06 & 6.69 & 3.72 & 6 & 0.8 \\
\hline 94.34 & 0.01 & 290.23 & 9.41 & 24.39 & 243.57 & 9.08 & 6.84 & 7 & 0.8 \\
\hline 94.03 & 0.02 & 290.22 & 9.69 & 23.54 & 241.85 & 9.31 & 5.43 & 8 & 0.8 \\
\hline 93.38 & 0.02 & 288.33 & 8.77 & 23.21 & 240.69 & 8.35 & 5.42 & 9 & 0.8 \\
\hline 92.60 & 0.02 & 289.14 & 10.56 & 24.19 & 240.79 & 9.03 & 6.02 & 10 & 0.8 \\
\hline
\end{tabular}

Again, shown are the percentage of target events detected (\%E), the average number of false events declared (\#FE), and the geo-location RMSEs (in metres). RMSEs are shown for all target geo-locations determined across the 1000 simulations (i.e. 0\% exclusions), and with the worst 1\% of geo-location estimates excluded from each RMSE calculation. Again, also shown are the percentage increase of the TALA RMSE over the gTALA RMSE (\% $\uparrow$ gTALA) and the percentage increase of the TALA RMSE over the CRLB RMSE (\%个 CRLB)

Deciding when it is necessary to make computational simplifications can be performed "on-the-fly", based on the number of measurements generated within the time window under consideration. A larger number of measurements may indicate a large number of emitters and/or signals that are closely spaced in time, which may then necessitate computational savings. Of course, the algorithmic complexity that can be afforded depends on the timecriticality of the application, specifically whether the com- mander requires the processing to be performed almost instantaneously (e.g. in order to immediately perform a retaliatory strike), or whether a longer processing time can be tolerated (e.g. when conducting post-event analysis).

\section{Future work}

1. To remind the reader, the TALA implementation herein requires that three heuristic parameters be set, these being (i): $\xi \in[0,1]$ in Eq. (3), (ii): $\mu \in[0,1]$ 
Table 6 TALA average computational time, and the average number of candidate locations at the end of each step of the TALA, for scenario 2

\begin{tabular}{|c|c|c|c|c|c|c|c|c|}
\hline \multirow{3}{*}{$\begin{array}{l}\text { TALA } \\
\text { run time } \\
\text { (s) }\end{array}$} & \multicolumn{6}{|c|}{ Average number of candidate positions } & \multirow{3}{*}{ Events } & \multirow{3}{*}{$P_{d}$} \\
\hline & Step 1 & Step 3 & Step 3 & Step 3 & Step 4 & Step 5 & & \\
\hline & & (crit 1) & (crit 2) & (crit 3) & & & & \\
\hline 0.01 & 20.60 & 8.14 & 4.00 & 0.98 & 0.98 & 0.98 & 1 & 1.0 \\
\hline 0.01 & 53.16 & 17.36 & 8.36 & 1.97 & 1.97 & 1.97 & 2 & 1.0 \\
\hline 0.02 & 95.94 & 27.17 & 13.19 & 2.94 & 2.94 & 2.93 & 3 & 1.0 \\
\hline 0.07 & 157.20 & 38.03 & 18.28 & 3.94 & 3.94 & 3.92 & 4 & 1.0 \\
\hline 0.14 & 224.86 & 50.44 & 23.96 & 4.91 & 4.91 & 4.89 & 5 & 1.0 \\
\hline 0.23 & 303.82 & 63.97 & 29.81 & 5.89 & 5.89 & 5.87 & 6 & 1.0 \\
\hline 0.42 & 397.65 & 75.00 & 35.38 & 6.88 & 6.88 & 6.85 & 7 & 1.0 \\
\hline 0.63 & 494.44 & 90.18 & 42.58 & 7.85 & 7.85 & 7.82 & 8 & 1.0 \\
\hline 0.98 & 602.77 & 103.05 & 48.20 & 8.84 & 8.84 & 8.81 & 9 & 1.0 \\
\hline 1.53 & 731.13 & 119.31 & 55.76 & 9.81 & 9.81 & 9.78 & 10 & 1.0 \\
\hline 0.00 & 17.70 & 6.64 & 3.24 & 0.97 & 0.97 & 0.97 & 1 & 0.9 \\
\hline 0.01 & 44.37 & 14.07 & 6.96 & 1.95 & 1.95 & 1.94 & 2 & 0.9 \\
\hline 0.02 & 82.47 & 22.38 & 11.20 & 2.92 & 2.92 & 2.91 & 3 & 0.9 \\
\hline 0.05 & 129.90 & 31.09 & 15.43 & 3.88 & 3.88 & 3.87 & 4 & 0.9 \\
\hline 0.11 & 186.77 & 41.34 & 20.24 & 4.85 & 4.85 & 4.83 & 5 & 0.9 \\
\hline 0.20 & 253.62 & 52.50 & 25.55 & 5.85 & 5.85 & 5.83 & 6 & 0.9 \\
\hline 0.34 & 330.56 & 62.03 & 30.28 & 6.79 & 6.79 & 6.76 & 7 & 0.9 \\
\hline 0.49 & 406.79 & 74.07 & 36.12 & 7.75 & 7.75 & 7.72 & 8 & 0.9 \\
\hline 0.73 & 500.82 & 85.82 & 41.60 & 8.72 & 8.72 & 8.70 & 9 & 0.9 \\
\hline 1.08 & 603.86 & 99.32 & 48.15 & 9.68 & 9.68 & 9.64 & 10 & 0.9 \\
\hline 0.00 & 15.82 & 5.38 & 2.77 & 0.95 & 0.95 & 0.94 & 1 & 0.8 \\
\hline 0.01 & 37.45 & 11.43 & 5.76 & 1.90 & 1.90 & 1.89 & 2 & 0.8 \\
\hline 0.01 & 68.19 & 17.84 & 9.18 & 2.87 & 2.87 & 2.85 & 3 & 0.8 \\
\hline 0.02 & 110.12 & 25.08 & 13.02 & 3.80 & 3.80 & 3.79 & 4 & 0.8 \\
\hline 0.09 & 158.49 & 33.88 & 17.42 & 4.76 & 4.76 & 4.74 & 5 & 0.8 \\
\hline 0.16 & 213.10 & 43.44 & 21.94 & 5.72 & 5.72 & 5.70 & 6 & 0.8 \\
\hline 0.24 & 273.47 & 50.27 & 25.60 & 6.65 & 6.65 & 6.61 & 7 & 0.8 \\
\hline 0.39 & 340.90 & 60.32 & 30.59 & 7.58 & 7.58 & 7.54 & 8 & 0.8 \\
\hline 0.52 & 415.07 & 69.45 & 35.09 & 8.45 & 8.45 & 8.42 & 9 & 0.8 \\
\hline 0.76 & 495.93 & 79.54 & 40.03 & 9.31 & 9.31 & 9.28 & 10 & 0.8 \\
\hline
\end{tabular}

used in deletion criterion 1 on Step 3, and (iii): $\gamma>0$ in Eq. (34). The performance of the TALA is also dependent on the measurement errors (i.e. $\sigma_{\theta}$ and $\sigma_{t}$ in the focal simulations). It would be interesting to analyse the performance of the TALA as a function of these parameters, in order to determine optimal values for the heuristic parameters as a function of the measurement errors. Such an analysis may offer valuable insight into how to optimise the performance of the TALA across a broad range of operational scenarios.
2. The performance of the TALA was compared to both the CRLB [23] and the performance of a "genie" TALA that exploited the true measurement-to-target associations. It would be interesting to compare the performance of the TALA to that of existing methods developed for specific applications, most notably the approaches of $[2,10,19,20]$ developed for multi-target localisation using TOA measurements and the approaches of $[4,5,11,12])$ developed in order to localise a single emitter using hybrid AOA/TDOA measurements. 
Table 7 Summary of the TALA geo-location performance for scenario 3

\begin{tabular}{|c|c|c|c|c|c|c|c|c|c|}
\hline \multicolumn{5}{|c|}{ TALA (0\% exclusions) } & \multicolumn{3}{|c|}{ TALA (1\% exclusions) } & \multirow{2}{*}{ Events } & \multirow{2}{*}{$P_{d}$} \\
\hline$\% \mathrm{E}$ & \#FE & RMSE & $\% \uparrow$ gTALA & $\% \uparrow C R L B$ & RMSE & $\% \uparrow$ gTALA & $\% \uparrow C R L B$ & & \\
\hline 98.80 & 0.00 & 322.48 & 1.35 & 13.32 & 288.98 & 2.88 & 3.74 & 1 & 1.0 \\
\hline 97.95 & 0.00 & 345.15 & 0.13 & 17.89 & 297.41 & 2.14 & 3.58 & 2 & 1. \\
\hline 97.07 & 0.01 & 361.52 & 5.67 & 25.05 & 290.89 & 3.33 & 2.52 & 3 & 1.0 \\
\hline 95.23 & 0.01 & 366.09 & 10.33 & 27.86 & 307.61 & 6.49 & 9.54 & 4 & 1. \\
\hline 94.52 & 0.01 & 379.98 & 9.55 & 32.95 & 310.85 & 9.98 & 10.97 & 5 & 1. \\
\hline 93.05 & 0.01 & 387.99 & 13.53 & 33.68 & 317.57 & 12.24 & 11.60 & 6 & 1.0 \\
\hline 93.01 & 0.02 & 390.64 & 10.55 & 35.78 & 313.90 & 10.25 & 11.27 & 7 & 1. \\
\hline 91.60 & 0.02 & 386.88 & 13.69 & 33.54 & 309.56 & 10.31 & 8.95 & 8 & 1.0 \\
\hline 91.17 & 0.03 & 406.21 & 16.30 & 40.65 & 325.19 & 11.81 & 14.79 & 9 & 1.0 \\
\hline 89.81 & 0.03 & 376.56 & 13.63 & 31.32 & 315.84 & 13.47 & 12.36 & 10 & 1.0 \\
\hline 98.10 & 0.00 & 386.89 & 6.51 & 24.38 & 320.99 & 6.38 & 6.05 & 1 & 0.9 \\
\hline 96.10 & 0.01 & 401.60 & 3.73 & 26.66 & 330.65 & 5.99 & 6.90 & 2 & 0.9 \\
\hline 95.63 & 0.01 & 394.15 & -1.23 & 25.29 & 327.46 & 3.16 & 7.42 & 3 & 0.9 \\
\hline 93.13 & 0.02 & 399.20 & 6.69 & 28.96 & 326.57 & 8.49 & 8.00 & 4 & 0.9 \\
\hline 92.18 & 0.01 & 421.33 & 6.91 & 36.20 & 339.06 & 6.76 & 12.38 & 5 & 0.9 \\
\hline 89.65 & 0.03 & 423.84 & 13.28 & 34.23 & 341.83 & 10.26 & 11.17 & 6 & 0.9 \\
\hline 90.30 & 0.03 & 446.99 & 17.38 & 43.35 & 350.90 & 12.17 & 15.52 & 7 & 0.9 \\
\hline 88.42 & 0.03 & 431.47 & 13.49 & 37.18 & 345.19 & 12.75 & 12.70 & 8 & 0.9 \\
\hline 87.40 & 0.04 & 453.87 & 19.29 & 44.99 & 360.63 & 15.51 & 18.17 & 9 & 0.9 \\
\hline 86.34 & 0.04 & 456.16 & 16.64 & 46.35 & 353.35 & 17.47 & 16.77 & 10 & 0.9 \\
\hline 94.00 & 0.01 & 421.12 & -5.11 & 23.97 & 359.77 & 4.31 & 10.18 & 1 & 0.8 \\
\hline 94.15 & 0.02 & 464.01 & 6.32 & 33.56 & 366.50 & 6.98 & 9.12 & 2 & 0.8 \\
\hline 91.73 & 0.02 & 473.74 & 11.72 & 36.06 & 377.14 & 9.41 & 12.75 & 3 & 0.8 \\
\hline 90.22 & 0.03 & 492.75 & 14.66 & 45.12 & 381.17 & 13.08 & 15.81 & 4 & 0.8 \\
\hline 88.86 & 0.04 & 504.91 & 12.79 & 47.65 & 392.40 & 13.57 & 18.91 & 5 & 0.8 \\
\hline 86.60 & 0.05 & 479.99 & 5.59 & 38.64 & 385.92 & 9.54 & 15.16 & 6 & 0.8 \\
\hline 86.80 & 0.06 & 486.42 & 20.15 & 41.21 & 382.79 & 15.62 & 15.09 & 7 & 0.8 \\
\hline 85.00 & 0.05 & 522.25 & 15.20 & 50.37 & 397.93 & 14.58 & 18.81 & 8 & 0.8 \\
\hline 83.92 & 0.07 & 506.22 & 11.31 & 47.08 & 401.54 & 17.14 & 20.90 & 9 & 0.8 \\
\hline 83.08 & 0.08 & 524.07 & 15.39 & 52.56 & 404.82 & 19.72 & 22.49 & 10 & 0.8 \\
\hline
\end{tabular}

Again, shown are the percentage of target events detected (\%E), the average number of false events declared (\#FE), and the geo-location RMSEs (in metres). RMSEs are shown for all target geo-locations determined across the 1000 simulations (i.e. $0 \%$ exclusions), and with the worst $1 \%$ of geo-location estimates excluded from each RMSE calculation. Again, also shown are the percentage increase of the TALA RMSE over the gTALA RMSE (\% $\uparrow$ gTALA) and the percentage increase of the TALA RMSE over the CRLB RMSE (\%个 CRLB)

3. The TALA implementation introduced herein implicitly assumes that the measurement error standard deviations are known. Extensive field testing of a prototype system may enable accurate error statistics to be determined, making this a valid assumption. In cases for which field testing is not practicable, or in extended operating conditions, measurement error standard deviations may not be accurately known. In such cases, careful setting of the standard deviations within the TALA is required.

Setting values too small (i.e. being overally optimistic as to the accuracy of each measurement) may inhibit data association, resulting in valid measurements being discarded. Conversely, setting values too high may fail to fully exploit accurate measurements. Analysis of this tradeoff is left for future work. 
Table 8 TALA average computational time, and the average number of candidate locations at the end of each step of the TALA, for scenario 3

\begin{tabular}{|c|c|c|c|c|c|c|c|c|}
\hline \multirow{3}{*}{$\begin{array}{l}\text { TALA } \\
\text { run time } \\
\text { (s) }\end{array}$} & \multicolumn{6}{|c|}{ Average number of candidate positions } & \multirow{3}{*}{ Events } & \multirow{3}{*}{$P_{d}$} \\
\hline & Step 1 & Step 3 & Step 3 & Step 3 & Step 4 & Step 5 & & \\
\hline & & (crit 1) & (crit 2) & (crit 3) & & & & \\
\hline 0.01 & 55.53 & 10.05 & 5.04 & 1.01 & 1.01 & 0.99 & 1 & 1.0 \\
\hline 0.01 & 97.69 & 22.35 & 11.86 & 2.01 & 1.99 & 1.96 & 2 & 1.0 \\
\hline 0.04 & 149.39 & 35.80 & 20.03 & 3.00 & 2.98 & 2.92 & 3 & 1.0 \\
\hline 0.03 & 215.67 & 53.84 & 31.83 & 3.94 & 3.92 & 3.82 & 4 & 1.0 \\
\hline 0.08 & 284.31 & 76.63 & 47.35 & 4.91 & 4.88 & 4.74 & 5 & 1.0 \\
\hline 0.12 & 368.60 & 102.52 & 65.49 & 5.83 & 5.79 & 5.59 & 6 & 1.0 \\
\hline 0.17 & 463.94 & 130.97 & 86.62 & 6.77 & 6.72 & 6.53 & 7 & 1.0 \\
\hline 0.24 & 563.94 & 171.32 & 116.61 & 7.69 & 7.63 & 7.35 & 8 & 1.0 \\
\hline 0.33 & 674.47 & 213.64 & 149.62 & 8.62 & 8.55 & 8.24 & 9 & 1.0 \\
\hline 0.43 & 803.40 & 267.18 & 191.98 & 9.48 & 9.40 & 9.01 & 10 & 1.0 \\
\hline 0.01 & 52.12 & 8.24 & 4.18 & 1.02 & 1.01 & 0.98 & 1 & 0.9 \\
\hline 0.02 & 89.31 & 17.86 & 9.72 & 1.99 & 1.96 & 1.93 & 2 & 0.9 \\
\hline 0.02 & 134.80 & 28.85 & 16.10 & 2.96 & 2.93 & 2.88 & 3 & 0.9 \\
\hline 0.04 & 187.96 & 42.65 & 25.40 & 3.88 & 3.83 & 3.74 & 4 & 0.9 \\
\hline 0.04 & 247.24 & 60.20 & 37.14 & 4.81 & 4.75 & 4.62 & 5 & 0.9 \\
\hline 0.13 & 318.68 & 79.85 & 51.03 & 5.67 & 5.58 & 5.40 & 6 & 0.9 \\
\hline 0.17 & 396.73 & 102.12 & 67.94 & 6.61 & 6.53 & 6.35 & 7 & 0.9 \\
\hline 0.24 & 476.01 & 129.76 & 88.87 & 7.46 & 7.38 & 7.11 & 8 & 0.9 \\
\hline 0.31 & 571.35 & 163.07 & 114.19 & 8.30 & 8.20 & 7.91 & 9 & 0.9 \\
\hline 0.41 & 674.19 & 204.32 & 147.04 & 9.18 & 9.07 & 8.67 & 10 & 0.9 \\
\hline 0.01 & 50.18 & 8.90 & 5.22 & 1.21 & 1.18 & 0.95 & 1 & 0.8 \\
\hline 0.01 & 81.14 & 19.47 & 11.79 & 2.18 & 2.14 & 1.90 & 2 & 0.8 \\
\hline 0.01 & 116.63 & 31.50 & 20.02 & 3.11 & 3.04 & 2.77 & 3 & 0.8 \\
\hline 0.03 & 162.80 & 48.00 & 31.80 & 4.05 & 3.97 & 3.64 & 4 & 0.8 \\
\hline 0.05 & 214.78 & 71.33 & 48.32 & 4.94 & 4.85 & 4.48 & 5 & 0.8 \\
\hline 0.11 & 272.45 & 95.83 & 66.88 & 5.82 & 5.70 & 5.25 & 6 & 0.8 \\
\hline 0.17 & 330.48 & 122.64 & 87.85 & 6.74 & 6.59 & 6.13 & 7 & 0.8 \\
\hline 0.20 & 401.28 & 160.11 & 116.92 & 7.60 & 7.45 & 6.85 & 8 & 0.8 \\
\hline 0.27 & 477.67 & 201.82 & 149.49 & 8.40 & 8.26 & 7.63 & 9 & 0.8 \\
\hline 0.36 & 564.86 & 250.12 & 188.52 & 9.28 & 9.11 & 8.38 & 10 & 0.8 \\
\hline
\end{tabular}

\section{Conclusions}

In this paper, a novel target acquisition and localisation algorithm (TALA) has been introduced that offers a capability for detecting and localising an unknown number of targets using the intermittent "signals-of-opportunity" they generate (e.g. "events" such as acoustic impulses or radio frequency transmissions). The TALA is a batch estimator, and its novelty lies in the mechanism by which it circumnavigates the need to perform global multi-sensor/multi-target data association (e.g. as necessary in [20]), which can be a computationally prohibitive combinatorial optimisation (e.g. see [22]). As a result, the TALA is able to maintain computational feasibility, even for large scale problems. Specifically, the TALA maintains a set of hypothesised target locations, and performs nearest neighbour data association on a measurementby-measurement basis, allowing each measurement to be associated with more than one hypothesised target 
location. The algorithm then determines maximum likelihood estimates of potential target locations, before a final downselection step ensures that each measurement is associated with no greater than one estimate.

The performance of the TALA is demonstrated for simulated scenarios with a network of 20 sensors and up to 10 targets. The sensors generate angle-of-arrival (AOA), time-of-arrival (TOA), or hybrid AOA/TOA measurements. Both simultaneous and non-simultaneous target events are considered, though clearly simultaneous events are more challenging, as the problem of resolving the association of measurements to events has greater ambiguity.

For non-simultaneous events, and with homogeneous sensors providing hybrid AOA/TOA measurements, the target localisation errors of the TALA are typically within $10-20 \%$ of an optimistic Cramér-Rao lower bound (CRLB) that ignores the multi-target data association problem. A better comparison shows that the localisation errors of the TALA are typically within 10\% of the errors generated by a "genie" algorithm that is given the correct measurement-to-target associations. Percentage differences in performance are reduced when a small percentage (i.e. 1\%) of outliers are removed from the comparisons, with the TALA RMSE typically then around 5\% greater than both the gTALA RMSE and the CRLB. For simultaneous events, again with sensors providing hybrid AOA/TOA measurements, there is only a few percent degradation in geolocation performance compared to the case of nonsimultaneous events. In both cases, the TALA successfully detect $93-98 \%$ of the targets, with virtually no false targets declared.

In the most difficult scenarios considered, with simultaneous events, and heterogeneous sensors providing either AOA or TOA measurements, the TALA continues to perform well in comparison to the gTALA and the optimistic CRLB. To elaborate, in this case, the RMSEs of the TALA and gTALA typically differ by $10-20 \%$, with the RMSE of the TALA typically $20-50 \%$ greater the CRLB. Again, when outliers are removed from the analysis, differences in performance are reduced significantly, with the TALA RMSE typically within $20 \%$ of the CRLB. The TALA detects $83-99 \%$ of the target events, whilst the average number of false events remains extremely low.

The computational expense of the TALA is shown to remain manageable as the number of targets increases. This allows the approach to be implemented in challenging time-critical scenarios, such as in the localisation of artillery firing events, for which there may be only a small window of opportunity in which to perform a retaliatory strike. It is concluded that the TALA provides a powerful situational awareness aid for passive surveillance operations.

\section{Endnotes}

${ }^{1}$ This is a common assumption in target state estimation problems (e.g. see [22]). For scenarios in which this assumption is violated, there are two options; either (i) a Gaussian approximation can be made or (ii) the likelihood functions and ML estimation approach within the TALA be modified in order to correctly account for the change in the measurement model.

${ }^{2}$ The maximum value of the individual likelihood is $l_{i}(\max )=\operatorname{det}\left(\boldsymbol{\Sigma}_{i}\right)^{-1 / 2} /(2 \pi)^{d_{i} / 2}$.

${ }^{3}$ Via experimentation, likelihood threshold values $\xi$ in the range $10^{-3}-10^{-2}$ were shown to generate excellent results.

${ }^{4}$ The GNSSE is the $\mathbb{R}^{3}$ vector: $\boldsymbol{F}\left(\boldsymbol{X}_{k}\right)^{\prime} \boldsymbol{\Sigma}^{-1}\left[\boldsymbol{Z}-\boldsymbol{f}\left(\boldsymbol{X}_{k}\right)\right]$. At the ML estimate, the GNSSE has a value of 0 .

${ }^{5}$ For example, if the measurement error covariance matrix $\boldsymbol{\Sigma}$ were block diagonal, it can easily be shown that

$\boldsymbol{F}\left(\boldsymbol{X}_{k}\right)^{\prime} \boldsymbol{\Sigma}^{-1}\left[\boldsymbol{Z}-\boldsymbol{f}\left(\boldsymbol{X}_{k}\right)\right]=\sum_{i=1}^{N_{a}} \boldsymbol{F}_{i}\left(\boldsymbol{X}_{k}\right)^{\prime} \boldsymbol{\Sigma}_{i}^{-1}\left[\boldsymbol{z}_{i}-\boldsymbol{f}\left(\boldsymbol{X}_{k} ; i\right)\right]$

where $\boldsymbol{F}_{i}\left(\boldsymbol{X}_{k}\right)^{\prime} \triangleq \nabla_{\boldsymbol{X}_{k}} \boldsymbol{f}\left(\boldsymbol{X}_{k} ; i\right)^{\prime}$. The complexity of the calculation of (41) is of order $\mathcal{O}\left(N_{a}\right)$. However, the calculation of $\boldsymbol{F}\left(\boldsymbol{X}_{k}\right)^{\prime} \boldsymbol{\Sigma}^{-1}\left[\boldsymbol{Z}-\boldsymbol{f}\left(\boldsymbol{X}_{k}\right)\right]$ using the full Hessian matrix $\boldsymbol{F}\left(\boldsymbol{X}_{k}\right)$ (given in Eq. (20)) and the full measurement error covariance matrix $\boldsymbol{\Sigma}$ (given in Eq. (13)) has complexity $\mathcal{O}\left(N_{a}^{3}\right)$.

\section{Appendix A: Determining the AOA and DDOA measurement intersections AOA intersections}

Consider two AOA measurements $\theta(i)$ generated by sensors $i=1,2$. It is straightforward to show that the point of intersection $\left(x_{I}, y_{I}\right)$ of these two measurements is given as follows:

$$
\begin{aligned}
& x_{I}=x_{S_{1}}+\frac{\Delta x \tan \theta(2)-\Delta y}{\tan \theta(2)-\tan \theta(1)} \\
& y_{I}=y_{S_{1}}+\frac{\Delta x \tan \theta(1) \tan \theta(2)-\Delta y \tan \theta(1)}{\tan \theta(2)-\tan \theta(1)}
\end{aligned}
$$

where $\Delta x$ and $\Delta y$ are the distances between the two sensors in the $x$ - and $y$-coordinate directions respectively, i.e.

$$
\begin{aligned}
& \Delta x=x_{S_{2}}-x_{S_{1}} \\
& \Delta y=y_{S_{2}}-y_{S_{1}}
\end{aligned}
$$

It is noted that Eqs. (42) - (43) will always output a value provided that the AOA measurements are not parallel (i.e. provided that $\theta(1) \neq \theta(2)$ and $\theta(1) \neq(\theta(2) \pm \pi))$. This 
is true even if the AOA measurements diverge, in which case the coordinates provided by Eqs. (42) - (43) will be in the opposite direction to at least one of the two measurements. Therefore, the point $\left(x_{I}, y_{I}\right)$ is only accepted as a valid intersection if $\theta(i)=\tan ^{-1}\left(\left(y_{I}-y_{S_{i}}\right) /\left(x_{I}-x_{S_{i}}\right)\right)$ for $i=1,2$.

\section{DDOA intersections}

Consider two DDOA measurements $\tau(R, i)$ at sensors $i=$ 1,2 . Let $X=(x, y)$ denote the point of intersection of these measurements. This point of intersection satisfies the following equations:

$$
\begin{aligned}
& h_{1}(\boldsymbol{X}) \triangleq\left[d_{R}(\boldsymbol{X})-d_{1}(\boldsymbol{X})-\tau(R, 1)\right]=0 \\
& h_{2}(\boldsymbol{X}) \triangleq\left[d_{R}(\boldsymbol{X})-d_{2}(\boldsymbol{X})-\tau(R, 2)\right]=0
\end{aligned}
$$

where $d_{i}(\boldsymbol{X})$ is again given by Eq. (29). Equations (46) and (47) each provide a hyperbola of potential solutions for $X$ (e.g. see Fig. 1), with two intersecting points. The intersection that is sought is the one that is in the direction of the AOA measurements. This intersection is determined using a N-R approach (e.g. see Section 9.6 in [25]). This is an iterative technique that generates a sequence of estimates $X_{k}, k=0,1,2, \ldots$ that will converge to a solution provided that the initial estimate $X_{0}$ is itself close enough to the intersection. In order to maximise the probability that the correct DDOA intersection will be determined, $\boldsymbol{X}_{0}$ is set to be the point of intersection of the two corresponding AOA measurements (if AOA measurements are also generated by the two sensors, and these measurements intersect). Otherwise, $X_{0}$ is set to be a location randomly generated within the surveillance region. Successive iterates of the N-R approach are given as follows:

$$
\boldsymbol{X}_{k+1}=\boldsymbol{X}_{k}-\boldsymbol{G}\left(\boldsymbol{X}_{k}\right)^{-1} \boldsymbol{h}\left(\boldsymbol{X}_{k}\right)
$$

where $\boldsymbol{h}\left(\boldsymbol{X}_{k}\right) \triangleq\left(h_{1}\left(\boldsymbol{X}_{k}\right) h_{2}\left(\boldsymbol{X}_{k}\right)\right)^{\prime}, \quad \boldsymbol{G}\left(\boldsymbol{X}_{k}\right)=$ $\left[\nabla_{\boldsymbol{X}_{k}}\left[\boldsymbol{h}\left(\boldsymbol{X}_{k}\right)^{\prime}\right]\right]^{\prime}$, and $\nabla_{\boldsymbol{X}_{k}}$ is the first-order partial derivative operator with respect to $\boldsymbol{X}_{k}$, i.e. $\nabla_{\boldsymbol{X}_{k}}=\left[\partial / \partial x_{k} \partial / \partial y_{k}\right]^{\prime}$. The matrix $\boldsymbol{G}\left(\boldsymbol{X}_{k}\right)$ is then given as follows:

$$
\boldsymbol{G}\left(\boldsymbol{X}_{k}\right)=\left(\begin{array}{ll}
\frac{\left(x_{k}-x_{S_{R}}\right)}{d_{R}\left(\boldsymbol{X}_{k}\right)}-\frac{\left(x_{k}-x_{S_{1}}\right)}{d_{1}\left(\boldsymbol{X}_{k}\right)} & \frac{\left(y_{k}-y_{S_{R}}\right)}{d_{R}\left(\boldsymbol{X}_{k}\right)}-\frac{\left(y_{k}-y_{S_{1}}\right)}{d_{1}\left(\boldsymbol{X}_{k}\right)} \\
\frac{\left(x_{k}-x_{S_{R}}\right)}{d_{R}\left(\boldsymbol{X}_{k}\right)}-\frac{\left(x_{k}-x_{S_{2}}\right)}{d_{2}\left(\boldsymbol{X}_{k}\right)} & \frac{\left(y_{k}-y_{S_{R}}\right)}{d_{R}\left(\boldsymbol{X}_{k}\right)}-\frac{\left(y_{k}-y_{S_{2}}\right)}{d_{2}\left(\boldsymbol{X}_{k}\right)}
\end{array}\right)
$$

A maximum of 100 iterations of the N-R approach are performed, and convergence to the DDOA intersection is assumed to have occurred if, on any iteration, the $x$ and $y$-coordinate location increments each have a magnitude smaller than $10^{-7}$. However, the N-R approach is not guaranteed to converge to the intersection, and when divergence occurs, further attempts are made to determine the DDOA intersection by re-initialising the N-R approach at a location randomly generated within the surveillance region. In the simulations presented herein, up to nine attempts are made using randomly generated initial locations.

\section{Competing interests}

The author declares that he/she has no competing interests.

\section{Publisher's Note}

Springer Nature remains neutral with regard to jurisdictional claims in published maps and institutional affiliations.

Received: 3 September 2016 Accepted: 3 May 2017

Published online: 25 May 2017

\section{References}

1. R Kaune, D Mušicki, W Koch, in Sensor Fusion and its Applications, ed. by C Thomas. On Passive Emitter Tracking in Sensor Networks (INTECH, Rijeka, 2010), pp. 293-318

2. G Simon, M Maróti, Á Lédeczi, G Balogh, B Kusy, A Nádas, G Pap, J Sallai, K Frampton, in Proceedings of the ACM 2nd International Conference on Embedded Networked Sensor Systems. Sensor Network-Based Countersniper System (ACM, New York, 2004), pp. 1-12

3. S Harman, V Calloway, N Wallace, A Hume, D Beale, in Proceedings of the IEEE Aerospace Conference, vol. 3. Sensor Network Performance Modeling for Weapon Locating (IEEE, Big Sky, 2004)

4. L Cong, W Zhuang, Hybrid TDOA/AOA mobile user location for wideband CDMA cellular systems. IEEE Trans.Wirel. Commun. 1(3), 439-447 (2002)

5. KW Cheung, MC So, W-K Ma, YT Chan, in EURASIP Journal on Applied Signal Processing. A constrained least squares approach to mobile positioning: algorithms and optimality (Hindawi Publishing Corporation, New York, 2006), pp. 1-23

6. D Mennitt, M Johns, Multiple-array passive acoustic source localization in urban environments. J. Acoust. Soc. Am. 127(5), 2932-2942 (2010)

7. K Pine, ME Evans, K Sammut, F He, in Proceedings of the 5 th Asian Control Conference, vol. 2. Radio direction finding for maritime search and rescue (IEEE, Melbourne, 2004), pp. 723-730

8. R Stansfield, Statistical Theory of DF Fixing. J. IEE IIIA Radiocommunication. 94(15), 762-770 (1947)

9. Y Bar-Shalom, X Li, T Kirubarajan, Estimation with Applications to Tracking and Navigation. (John Wiley and Sons Inc., New York, 2001)

10. S Venkateswaran, U Madhow, Localizing multiple events using times of arrival: a parallelized, hierarchical approach to the association problem. IEEE Trans. Signal Proc. 60(10), 5464-5477 (2012)

11. A Urruela, A Pages-Zamora, J Riba, in IEEE International Conference on Acoustics, Speech and Signal Processing (ICASSP), vol. 4. Divide-and-conquer based closed-form position estimation for AOA and TDOA measurements (IEEE, Toulouse, 2006)

12. AN Bishop, B Fidan, K Doǧançay, BDO Anderson, PN Pathirana, in Signal Processing, vol. 88. Exploiting geometry for improved hybrid AOA/TDOAbased localization (Elsevier, Amsterdam, 2008), pp. 1775-1791

13. X Sheng, Y-H Hu, Maximum likelihood multiple-source localization using acoustic energy measurements with wireless sensor networks. IEEE Trans. Signal Proc. 53(1), 44-53 (2005)

14. BK Chalise, YD Zhang, MG Amin, B Himed, Target localization in a multistatic passive radar system through convex optimization. Sig. Proc. 102 207-215 (2014)

15. M Alam, K Jamil, in IEEE Radar Conference. Maximum likelihood (ML) based localization algorithm for multi-static passive radar using range-only measurements (IEEE, Johannesburg, 2015), pp. 180-184

16. KYang, G Wang, ZQ Luo, Efficient convex relaxation methods for robust target localization by a sensor network using time differences of arrivals. IEEE Trans. Sig. Proc. 57(7), 2775-2784 (2009)

17. HWL Naus, CV van Wijk, Simultaneous localisation of multiple emitters. IEE Proc. Radar Sonar Navig. 151(2), 65-70 (2004)

18. AN Bishop, PN Pathirana, Localization of emitters via the intersection of bearing lines: a ghost elimination approach. IEEE Trans. Veh. Technol. 56(5), 3106-3110 (2007)

19. Y Lee, TS Wada, B-H Juang, in Proceedings of the IEEE International Conference on Acoustics, Speech and Signal Processing (ICASSP 10). Multiple 
acoustic source localization based on multiple hypotheses testing using particle approach (IEEE, Dallas, 2010), pp. 2722-2725

20. H Shen, Z Ding, S Dasgupta, C Zhao, Multiple source localization in wireless sensor networks based on time of arrival measurement. IEEE Trans. Sig. Proc. 62(8), 1938-1949 (2014)

21. S Blackman, R Popoli, Design and Analysis of Modern Tracking Systems. (Artech House, Norwood, Massachusets, 1999)

22. Y Bar-Shalom (ed.), Multitarget-Multisensor Tracking: Advanced Applications (Artech House, Norwood, Massachusets, 1990)

23. H Van Trees, Detection, Estimation, and Modulation Theory. (John Wiley and Sons Inc., New York, 1968)

24. A Björck, Numerical Methods for Least Squares Problems. (Society for Industrial and Applied Mathematics, Philadelphia, 1996)

25. WH Press, SA Teukolsky, WT Vetterling, BP Flannery, Numerical Recipes in C, The Art of Scientific Computing, 2nd edn. (Cambridge University Press, New York, 1992)

26. K Levenberg, A method for the solution of certain non-linear problems in least squares. Q. Appl. Math. 2, 164-168 (1944)

27. DW Marquardt, An algorithm for least-squares estimation of nonlinear parameters. J. Soc. Ind. Appl. Math. 1(2), 431-441 (1963)

28. JE Cavanaugh, RH Shumway, On computing the expected Fisher information matrix for state-space model parameters. Stat. Probab. Lett. 26(4), 347-355 (1996)

29. A Farina, B Ristic, L Timmoneri, Cramér-Rao bound for nonlinear filtering with $P_{d}<1$ and its application to target tracking. IEEE Trans. Sig. Proc. 50(8), 1916-1924 (2002)

30. ML Hernandez, B Ristic, A Farina, L Timmoneri, A comparison of two Cramér-Rao bounds for non-linear filtering with $P_{d}<1$. IEEE Trans. Sig. Proc. 52(9), 2361-2370 (2004)

31. R Tharmarasa, T Kirubarajan, ML Hernandez, A Sinha, PCRLB Based Multisensor array management for multitarget tracking. IEEE Trans. Aerosp. Electron. Syst. 43(2), 539-555 (2007)

32. ML Hernandez, A Farina, B Ristic, PCRLB for tracking in cluttered environments: measurement sequence conditioning approach. IEEE Trans. Aerosp. Electron. Syst. 42(2), 680-704 (2006)

33. Special issue on time delay estimation. IEEE Acoust. Speech Signal Process. Newsl. 49(1), 12-12 (1980). IEEE Journals \& Magazines

34. R Kaune, in Proceedings of the 15th International Conference on Information Fusion. accuracy studies for TDOA and TOA localization. (Singapore, 2012), pp. 408-415

\section{Submit your manuscript to a SpringerOpen ${ }^{\circ}$ journal and benefit from:}

- Convenient online submission

- Rigorous peer review

- Immediate publication on acceptance

- Open access: articles freely available online

- High visibility within the field

- Retaining the copyright to your article

Submit your next manuscript at $\gg$ springeropen.com 